\title{
An Efficient, Inexpensive and Shelf-Stable Diazotransfer Reagent: Imidazole-1-sulfonyl Azide Hydrochloride
}

\author{
Ethan D. Goddard-Borger* and Robert V. Stick \\ Chemistry M313, School of Biomedical, Biomolecular \& Chemical Sciences, \\ The University of Western Australia, Crawley WA 6009, Australia
}

\section{SUPPORTING INFORMATION}

\section{Experimental Section}

General experimental procedures have been given previously. ${ }^{1}$

\section{Other General Procedures}

\section{Procedure A - Preparation of azides (with a subsequent acetylation)}

Imidazole-1-sulfonyl azide hydrochloride $\mathbf{1 . H C l}(0.25 \mathrm{~g}, 1.2 \mathrm{mmol})$ was added to the amine or ammonium salt substrate $(1.0 \mathrm{mmol}), \mathrm{K}_{2} \mathrm{CO}_{3}(\mathrm{n}+0.5 \mathrm{mmol})^{2}$ and $\mathrm{CuSO}_{4} .5 \mathrm{H}_{2} \mathrm{O}(2.5 \mathrm{mg}, 10 \mu \mathrm{mol})$ in $\mathrm{MeOH}(5 \mathrm{~mL})$ and the mixture stirred at room temperature for the specified time (Table 1$){ }^{3}$ The mixture was concentrated and co-evaporated with $\mathrm{PhMe}(2 \times 10 \mathrm{~mL})$. Acetic anhydride $(0.76 \mathrm{~mL}, 8.0$ mmol) was added to the residue in $\mathrm{C}_{5} \mathrm{H}_{5} \mathrm{~N}(5 \mathrm{~mL})$ and the mixture stirred $(3 \mathrm{~h}$.). The mixture was concentrated, diluted with $\mathrm{H}_{2} \mathrm{O}(20 \mathrm{~mL})$ and extracted with EtOAc $(3 \times 15 \mathrm{~mL})$. The combined organic layers were dried $\left(\mathrm{MgSO}_{4}\right)$, filtered and concentrated. Flash chromatography gave the azide.

\section{Procedure B - Preparation of azides}

Imidazole-1-sulfonyl azide hydrochloride $\mathbf{1 . H C l}(0.25 \mathrm{~g}, 1.2 \mathrm{mmol})$ was added to the amine or ammonium salt substrate $(1.0 \mathrm{mmol}), \mathrm{K}_{2} \mathrm{CO}_{3}(\mathrm{n}+0.5 \mathrm{mmol})^{2}$ and $\mathrm{CuSO}_{4} .5 \mathrm{H}_{2} \mathrm{O}(2.5 \mathrm{mg}, 10 \mu \mathrm{mol})$ in $\mathrm{MeOH}(5 \mathrm{~mL})$ and the mixture stirred at room temperature for the specified time (Table 1$){ }^{3}$ The mixture was concentrated, diluted with $\mathrm{H}_{2} \mathrm{O}(15 \mathrm{~mL})$, acidified with conc. $\mathrm{HCl}$ and extracted with EtOAc $(3 \times 10 \mathrm{~mL})$. The combined organic layers were dried $\left(\mathrm{MgSO}_{4}\right)$, filtered and concentrated. Flash chromatography gave the azide.

\section{Procedure C - Preparation of diazo compounds}

Imidazole-1-sulfonyl azide hydrochloride $\mathbf{1 . H C l}(0.25 \mathrm{~g}, 1.2 \mathrm{mmol})$ was added to the substrate $(1.0$ mmol) and the specified base $(5.0 \mathrm{mmol})$ in $\mathrm{MeCN}(5 \mathrm{~mL})$ and the mixture stirred at $40^{\circ} \mathrm{C}$ for the specified time (Table 2). ${ }^{3}$ The mixture was diluted with EtOAc $(15 \mathrm{~mL})$, washed with hydrochloric acid $(2 \times 15 \mathrm{~mL}, 1 \mathrm{M}), \mathrm{H}_{2} \mathrm{O}(15 \mathrm{~mL})$, dried $\left(\mathrm{MgSO}_{4}\right)$, filtered and concentrated. Flash chromatography gave the diazo compound.

(1) Scaffidi, A.; Skelton, B. W.; Stick, R. V.; White, A. H. Aust. J. Chem. 2006, 59, 426-433.

(2) Where ' $n$ ' is the number of mols of acid in the system. For example: the reaction of $\mathbf{1 . H C l}(1.2 \mathrm{mmol})$ and the amino

acid L-valine $(1.0 \mathrm{mmol})$ requires $\mathrm{n}=2.2 \mathrm{mmol}$.

(3) Please refer to the Tables held within the paper. 


\section{Imidazole-1-sulfonyl Azide 1}

Sulfuryl chloride $(0.40 \mathrm{~mL}, 5.0 \mathrm{mmol})$ was added drop-wise to an ice-cooled suspension of $\mathrm{NaN}_{3}(0.32$ $\mathrm{g}, 5.0 \mathrm{mmol})$ in $\mathrm{MeCN}(5 \mathrm{~mL})$ and the mixture stirred overnight at room temperature. Imidazole $(0.68$ $\mathrm{g}, 10 \mathrm{mmol}$ ) was added to the ice-cooled mixture and the resulting slurry stirred for $3 \mathrm{~h}$. at room temperature. The mixture was diluted with EtOAc $(10 \mathrm{~mL})$ and $\mathrm{H}_{2} \mathrm{O}(10 \mathrm{~mL})$ and the aqueous layer separated and discarded. The organic layer was washed with $\mathrm{H}_{2} \mathrm{O}(10 \mathrm{~mL})$ then saturated aqueous $\mathrm{NaHCO}_{3}(2 \times 15 \mathrm{~mL})$, dried over $\mathrm{MgSO}_{4}$ and filtered. Concentration of the filtrate and flash chromatography (EtOAc/petrol, 1:3) gave 1 as a colourless liquid (0.62 g, 72\%). IR $v_{\max .}$ (film) 2171, 1387 and $1172 \mathrm{~cm}^{-1} .{ }^{1} \mathrm{H}$ NMR $\left(600 \mathrm{MHz}, \mathrm{CDCl}_{3}\right) \delta=7.17$ (dd, $\left.1 \mathrm{H}, J=0.8,1.7 \mathrm{~Hz}, \mathrm{H}-4\right), 7.35$ (dd, 1 $\mathrm{H}, J=1.4,1.7 \mathrm{~Hz}, \mathrm{H}-5), 7.96(\mathrm{dd}, 1 \mathrm{H}, J=0.8,1.4 \mathrm{~Hz}, \mathrm{H}-2) ;{ }^{13} \mathrm{C} \mathrm{NMR}\left(150.9 \mathrm{MHz}, \mathrm{CDCl}_{3}\right) \delta=117.7$ (C-5), 131.9 (C-4), 136.7 (C-2). HRMS (EI): $\mathrm{m} / \mathrm{z}=173.0013$; [M ] ${ }^{+\bullet}$ requires 173.0010. Anal. Calcd. For $\mathrm{C}_{3} \mathrm{H}_{3} \mathrm{~N}_{5} \mathrm{O}_{2} \mathrm{~S}$ : C, 20.81; H, 1.75; N, 40.45. Found: C, 20.93; H, 1.79; N, 40.22.

\section{Imidazole-1-sulfonyl Azide Hydrochloride 1.HCl}

Sulfuryl chloride $(16.1 \mathrm{~mL}, 200 \mathrm{~mol})$ was added drop-wise to an ice-cooled suspension of $\mathrm{NaN}_{3}$ (13.0 $\mathrm{g}, 200 \mathrm{mmol})$ in $\mathrm{MeCN}(200 \mathrm{~mL})$ and the mixture stirred overnight at room temperature. Imidazole $(25.9 \mathrm{~g}, 380 \mathrm{mmol})$ was added portion-wise to the ice-cooled mixture and the resulting slurry stirred for $3 \mathrm{~h}$. at room temperature. The mixture was diluted with EtOAc $(400 \mathrm{~mL})$, washed with $\mathrm{H}_{2} \mathrm{O}(2 \times 400$ $\mathrm{mL})$ then saturated aqueous $\mathrm{NaHCO}_{3}(2 \times 400 \mathrm{~mL})$, dried over $\mathrm{MgSO}_{4}$ and filtered. A solution of $\mathrm{HCl}$ in $\mathrm{EtOH}$ [obtained by the drop-wise addition of $\mathrm{AcCl}(21.3 \mathrm{~mL}, 300 \mathrm{mmol})$ to ice-cooled dry ethanol $(75 \mathrm{~mL})$ ] was added drop-wise to the filtrate with stirring, the mixture chilled in an ice-bath, filtered and the filter cake washed with EtOAc $(3 \times 100 \mathrm{~mL})$ to give $\mathbf{1 . H C l}$ as colourless needles $(26.4 \mathrm{~g}, 63 \%)$, m.p. $100-102^{\circ} \mathrm{C}^{4} \mathrm{IR} v_{\max }(\mathrm{KBr}) 2173,1384$ and $1161 \mathrm{~cm}^{-1} .{ }^{1} \mathrm{H}$ NMR $\left(600 \mathrm{MHz}, \mathrm{D}_{2} \mathrm{O}\right) \delta=7.68(\mathrm{dd}, 1$ $\mathrm{H}, J=1.3,2.2 \mathrm{~Hz}, \mathrm{H}-4), 8.09$ (dd, $1 \mathrm{H}, J=1.6,2.2 \mathrm{~Hz}, \mathrm{H}-5$ ), 9.53 (dd, $1 \mathrm{H}, J=1.3,1.6 \mathrm{~Hz}, \mathrm{H}-2) ;{ }^{13} \mathrm{C}$ NMR $\left(150.9 \mathrm{MHz}, \mathrm{D}_{2} \mathrm{O}\right) \delta=120.8,123.4,138.3$. HRMS (FAB): $\mathrm{m} / \mathrm{z}=174.0072 ;\left[\mathrm{M}-\mathrm{Cl}^{+}\right.$requires 174.0081. Anal. Calcd. For $\mathrm{C}_{3} \mathrm{H}_{4} \mathrm{ClN}_{5} \mathrm{O}_{2} \mathrm{~S}$ : C, 17.19; H, 1.92; N, 33.41. Found: C, 17.30; H, 1.99; N, 33.13 .

\section{1,3,4,6-Tetra-O-acetyl-2-azido-2-deoxy-D-glucose}

1) D-Glucosamine hydrochloride $(216 \mathrm{mg}, 1.00 \mathrm{mmol})$ was treated according to Procedure A, with the use of $\mathbf{1}(1.20 \mathrm{mmol})$ instead of $\mathbf{1 . H C l}$ [flash chromatography (EtOAc/petrol, 1:3)], to give 1,3,4,6tetra- $O$-acetyl-2-azido-2-deoxy-D-glucose as a colourless gum (343 mg, 92\%). The IR, ${ }^{1} \mathrm{H}$ and ${ }^{13} \mathrm{C}$ NMR spectroscopic data were in agreement with those published. ${ }^{5}$ Anal. Calcd. For $\mathrm{C}_{14} \mathrm{H}_{19} \mathrm{~N}_{3} \mathrm{O}_{9}$ : C, 45.04; H, 5.13; N, 11.26. Found: C, 45.19; H, 5.04; N, 11.07.

2) D-Glucosamine hydrochloride $(10.8 \mathrm{~g}, 50.0 \mathrm{mmol})$ was treated according to Procedure A [flash chromatography (EtOAc/petrol, 1:3)] to give 1,3,4,6-tetra- $O$-acetyl-2-azido-2-deoxy-D-glucose as a colourless gum $(17.2 \mathrm{~g}, 92 \%)$.

(4) The evolution of gas from the melt of 1.HCl suggests decomposition upon melting - see the DSC trace on page six. Please note that DSC on an identical sample suggests a melting point of $94^{\circ} \mathrm{C}$, different to the $100-102^{\circ} \mathrm{C}$ obtained on a hot stage melting apparatus.

(5) Vasella, A.; Witzig, C.; Chiara, J.-L.; Martin-Lomas, M. Helv. Chim. Acta 1991, 74, 2073-2077. 


\section{1,3,4,6-Tetra-O-acetyl-2-azido-2-deoxy-D-galactose}

D-Galactosamine hydrochloride $(108 \mathrm{mg}, 0.500 \mathrm{mmol})$ was treated according to Procedure A [flash chromatography (EtOAc/petrol, 1:3)] to give 1,3,4,6-tetra-O-acetyl-2-azido-2-deoxy-D-galactose as a colourless gum (162 mg, 87\%).The IR, ${ }^{1} \mathrm{H}$ and ${ }^{13} \mathrm{C}$ NMR spectroscopic data were in agreement with those published. ${ }^{5}$ Anal. Calcd. For $\mathrm{C}_{14} \mathrm{H}_{19} \mathrm{~N}_{3} \mathrm{O}_{9}$ : C, 45.04; H, 5.13; N, 11.26. Found: C, 45.28; H, 5.28; N, 11.11.

\section{(2S)-2-Azido-3-methylbutanoic acid}

L-Valine $(117 \mathrm{mg}, 1.00 \mathrm{mmol})$ was treated according to Procedure B [flash chromatography (EtOAc/petrol/AcOH, 20:79:1)] to give (2S)-2-azido-3-methylbutanoic acid as a pale yellow oil (120mg, 84\%). $[\alpha]_{\mathrm{D}}=-46.5\left(\mathrm{c}=1.0\right.$ in $\mathrm{CHCl}_{3}$, lit. $\left.^{6}[\alpha]_{\mathrm{D}}=-47.8\right)$. The ${ }^{1} \mathrm{H}$ and ${ }^{13} \mathrm{C}$ NMR spectroscopic data were in agreement with those published. ${ }^{6}$

\section{(2S)-2-Azido-4-methylpentanoic acid}

L-Leucine (131 mg, $1.00 \mathrm{mmol}$ ) was treated according to Procedure B [flash chromatography (EtOAc/petrol/AcOH, 15:84:1)] to give (2S)-2-azido-4-methylpentanoic acid as a pale yellow oil (134 mg, 85\%). $[\alpha]_{\mathrm{D}}=-13.8\left(\mathrm{c}=1.0\right.$ in $\mathrm{CHCl}_{3}$, lit. $\left.^{6}[\alpha]_{\mathrm{D}}=-13.0\right)$. The ${ }^{1} \mathrm{H}$ and ${ }^{13} \mathrm{C}$ NMR spectroscopic data were in agreement with those published. ${ }^{6}$

\section{(2S)-2-Azido-3-phenylpropanoic Acid}

L-Phenylalanine (165 mg, $1.00 \mathrm{mmol}$ ) was treated according to Procedure B [flash chromatography (EtOAc/petrol/AcOH, 10:89:1)] to give (2S)-2-azido-3-phenylpropanoic acid as a pale yellow oil (143 $\mathrm{mg}, 75 \%)$. $[\alpha]_{\mathrm{D}}=-72.1\left(\mathrm{c}=1.0\right.$ in $\mathrm{CHCl}_{3}$, lit. $\left.^{6}[\alpha]_{\mathrm{D}}=-74.2\right)$. The ${ }^{1} \mathrm{H}$ and ${ }^{13} \mathrm{C}$ NMR spectroscopic data were in agreement with those published. ${ }^{6}$ Anal. Calcd. For $\mathrm{C}_{9} \mathrm{H}_{9} \mathrm{~N}_{3} \mathrm{O}_{2}$ : C, 56.54; H, 4.74; N, 21.98. Found: C, 56.45; H, 4.92; N, 21.74.

\section{(2S)-2,6-Diazidohexanoic Acid}

L-Lysine hydrochloride (183 mg, $1.00 \mathrm{mmol}$ ) was treated according to Procedure B, with the addition of $\mathrm{H}_{2} \mathrm{O}(5 \mathrm{~mL})$ as a co-solvent [flash chromatography (EtOAc/petrol/AcOH, 10:89:1)], to give (2S)2,6-diazidohexanoic acid as a pale yellow oil (131 mg, 66\%). $[\alpha]_{\mathrm{D}}=-49.3$. IR $v_{\max }$. (film) 2178,1683 $\mathrm{cm}^{-1} .{ }^{1} \mathrm{H}$ NMR (600 MHz, $\mathrm{CDCl}_{3}$ ) $\delta=1.50-1.68$ (m, $\left.4 \mathrm{H}, \mathrm{H}-4,5\right), 1.78-1.95$ (m, $2 \mathrm{H}, \mathrm{H}-3$ ), 3.30-3.33 (m, $2 \mathrm{H}, \mathrm{H}-6), 3.95$ (dd, $1 \mathrm{H}, J=5.1,8.4 \mathrm{~Hz}, \mathrm{H}-2) ;{ }^{13} \mathrm{C} \mathrm{NMR}\left(150.9 \mathrm{MHz}, \mathrm{CDCl}_{3}\right) \delta=23.1(\mathrm{C}-4), 28.5$ (C-5), 31.0 (C-3), 51.2 (C6), 61.7 (C2), 175.2 (C-1). HRMS (EI): m/z = 198.0883; [M] $]^{\bullet}$ requires 198.0871. Anal. Calcd. For $\mathrm{C}_{6} \mathrm{H}_{10} \mathrm{~N}_{6} \mathrm{O}_{2}$ : C, 36.36; H, 5.09; N, 42.41. Found: C, 36.21; H, 5.28; N, 42.30 .

(6) Lundquist, IV, J. T.; Pelletier, J. C. Org. Lett. 2001, 3, 781-783. 


\section{4-Azidobutanoic Acid}

4-Aminobutanoic acid $(103 \mathrm{mg}, 1.00 \mathrm{mmol})$ was treated according to Procedure B [flash chromatography (EtOAc/petrol/AcOH, 5:94:1)] to give 4-azidobutanoic acid as a pale yellow oil (93 $\mathrm{mg}, 72 \%$ ). The IR, ${ }^{1} \mathrm{H}$ and ${ }^{13} \mathrm{C}$ NMR spectroscopic data were in agreement with those published. ${ }^{7}$

\section{Methyl (2S)-2-Azido-3-(4-hydroxyphenyl)propanoate}

L-Tyrosine methyl ester hydrochloride $(232 \mathrm{mg}, 1.00 \mathrm{mmol}$ ) was treated according to Procedure B [flash chromatography (EtOAc/petrol, 1:3)] to give methyl (2S)-2-azido-3-(4hydroxyphenyl)propanoate as a pale yellow oil (199 mg, 90\%), $[\alpha]_{\mathrm{D}}=-10.3$. IR $v_{\max }$ (film) 2172,1691 $\mathrm{cm}^{-1} .{ }^{1} \mathrm{H}$ NMR $\left(600 \mathrm{MHz}, \mathrm{CDCl}_{3}\right) \delta=2.95(\mathrm{dd}, 1 \mathrm{H}, J=8.5,14.1 \mathrm{~Hz}, \mathrm{H}-3), 3.10(\mathrm{dd}, 1 \mathrm{H}, J=5.4,14.1$ Hz, H-3), 3.77 (s, $3 \mathrm{H}, \mathrm{OCH}_{3}$ ), 4.02 (dd, $\left.1 \mathrm{H}, J=5.4,8.5 \mathrm{~Hz}, \mathrm{H}-2\right), 4.88$ (s, $\left.1 \mathrm{H}, \mathrm{OH}\right), 6.77-6.80$ (m, 2 $\mathrm{H}, \mathrm{ArH}), 7.09-7.11(\mathrm{~m}, 2 \mathrm{H}, \mathrm{ArH}){ }^{13} \mathrm{C} \mathrm{NMR}\left(150.9 \mathrm{MHz}, \mathrm{CDCl}_{3}\right) \delta=37.0(\mathrm{C}-3), 52.8\left(\mathrm{OCH}_{3}\right), 63.6$ (C-2), 115.7-155.0 (Ar), 170.6 (C-1). HRMS (FAB): $\mathrm{m} / \mathrm{z}=222.0901$; $[\mathrm{M}+\mathrm{H}]^{+}$requires 222.0888. Anal. Calcd. For $\mathrm{C}_{10} \mathrm{H}_{11} \mathrm{~N}_{3} \mathrm{O}_{3}$ : C, 54.29; H, 5.01; N, 19.00. Found: C, 54.35; H, 5.18; N, 18.79 .

\section{Methyl (2S)-2-Azido-3-hydroxypropanoate}

L-Serine methyl ester hydrochloride $(156 \mathrm{mg}, 1.00 \mathrm{mmol}$ ) was treated according to Procedure B [flash chromatography (EtOAc/petrol, 2:3)] to give methyl (2S)-2-azido-3-hydroxypropanoate as a pale yellow oil $(120 \mathrm{mg}, 83 \%),[\alpha]_{\mathrm{D}}=-93.4\left(\mathrm{c}=1.0\right.$ in $\mathrm{CHCl}_{3}$, lit. $\left.^{8}[\alpha]_{\mathrm{D}}=-92.2\right)$. The ${ }^{1} \mathrm{H}$ and ${ }^{13} \mathrm{C} \mathrm{NMR}$ spectroscopic data were in agreement with those published. ${ }^{8}$ Anal. Calcd. For $\mathrm{C}_{4} \mathrm{H}_{7} \mathrm{~N}_{3} \mathrm{O}_{3}: \mathrm{C}, 33.11 ; \mathrm{H}$, 4.86; N, 28.96. Found: C, 32.90; H, 4.89; N, 28.82.

\section{(1S,2S)-2-Azido-1-phenyl-1,3-propanediol}

(1S,2S)-2-Amino-1-phenyl-1,3-propanediol $(167 \mathrm{mg}, 1.00 \mathrm{mmol})$ was treated according to Procedure B [flash chromatography (EtOAc/petrol, 1:3)] to give $(1 S, 2 S)$-2-azido-1-phenyl-1,3-propanediol as a pale yellow oil $(176 \mathrm{mg}, 91 \%),[\alpha]_{\mathrm{D}}=-72.0\left(\mathrm{c}=1.0\right.$ in $\mathrm{CHCl}_{3}$, lit. $\left.{ }^{9}[\alpha]_{\mathrm{D}}=-76.26\right)$. The IR, ${ }^{1} \mathrm{H}$ and ${ }^{13} \mathrm{C}$ NMR spectroscopic data were in agreement with those published. ${ }^{9}$

\section{4-Azido-1-methoxybenzene}

4-Anisidine (123 mg, $1.00 \mathrm{mmol}$ ) was treated according to Procedure B [flash chromatography (EtOAc/petrol, 1:3)] to give 4-azido-1-methoxybenzene as a yellow oil (116 mg, 78\%). The IR, ${ }^{1} \mathrm{H}$ and ${ }^{13} \mathrm{C}$ NMR spectroscopic data were in agreement with those published. ${ }^{10}$

(7) van der Peet, P.; Gannon, C. T.; Walker, I.; Dinev, Z.; Angelin, M.; Tam, S.; Ralton, J. E.; McConville, M. J.; Williams, S. J. ChemBioChem 2006, 7, 1384-1391.

(8) Manabe, S.; Sakamoto, K.; Nakahara, Y.; Sisido, M.; Hohsaka, T.; Ito, Y. Bioorg. Med. Chem. 2002, 10, 573-581.

(9) Hajura, S; Karmakar, A.; Maji, T.; Medda, A. K. Tetrahedron 2006, 62, 8959-1965.

(10) Liu, Q.; Tor, Y. Org. Lett. 2003, 5, 2571-2572. 


\section{4-Azidobenzoic acid}

4-Aminobenzoic acid (137 mg, $1.00 \mathrm{mmol})$ was treated according to Procedure B [flash chromatography (EtOAc/petrol/AcOH, 25:72:3)] to give 4-azidobenzoic acid as pale yellow crystals (139, 85\%), m.p. $188.5-190^{\circ} \mathrm{C}$ (lit. ${ }^{11}$ m.p. $\left.188-191{ }^{\circ} \mathrm{C}\right)$. The IR, ${ }^{1} \mathrm{H}$ and ${ }^{13} \mathrm{C}$ NMR spectroscopic data were in agreement with those published. ${ }^{10}$

\section{2-Azidobenzoic acid}

2-Aminobenzoic acid (137 mg, $1.00 \mathrm{mmol})$ was treated according to Procedure B [flash chromatography (EtOAc/petrol/AcOH, 25:72:3)] to give 2-azidobenzoic acid as pale yellow crystals (135 mg, 83\%), m.p. $147-148.5^{\circ} \mathrm{C}$ (lit. ${ }^{11}$ m.p. $146-148^{\circ} \mathrm{C}$ ). The IR and ${ }^{1} \mathrm{H}$ NMR spectroscopic data were in agreement with those published. ${ }^{11}$

\section{Diethyl Diazomalonate}

Diethyl malonate $(0.15 \mathrm{~mL}, 1.0 \mathrm{mmol})$ was treated according to Procedure $\mathrm{C}$, using $\mathrm{K}_{2} \mathrm{CO}_{3}(0.69 \mathrm{~g}, 5.0$ $\mathrm{mmol}$ ) as base [flash chromatography (EtOAc/petrol, 1:19)], to give diethyl diazomalonate as a yellow oil $(0.12 \mathrm{~g}, 65 \%)$. The IR and ${ }^{1} \mathrm{H}$ NMR spectroscopic data were in agreement with those published. ${ }^{12}$ HRMS (FAB): $\mathrm{m} / \mathrm{z}=187.0714 ;[\mathrm{M}+\mathrm{H}]^{+}$requires 187.0719. Anal. Calcd. For $\mathrm{C}_{7} \mathrm{H}_{10} \mathrm{~N}_{2} \mathrm{O}_{4}: \mathrm{C}, 45.16$; H, 5.41; N, 15.05. Found: C, 45.42; H, 5.23; N, 14.93 .

\section{Ethyl 2-Diazoacetoacetate}

Ethyl acetoacetate $(0.13 \mathrm{~mL}, 1.0 \mathrm{mmol})$ was treated according to Procedure $\mathrm{C}$, using $\mathrm{K}_{2} \mathrm{CO}_{3}(0.69 \mathrm{~g}$, $5.0 \mathrm{mmol}$ ) as base [flash chromatography (EtOAc/petrol, 1:19)], to give ethyl 2-diazoacetoacetate as a yellow oil (92 mg, 59\%). The IR and ${ }^{1} \mathrm{H}$ spectroscopic data were in agreement with those published. ${ }^{12}$ HRMS (FAB): $\mathrm{m} / \mathrm{z}=157.0617 ;[\mathrm{M}+\mathrm{H}]^{+}$requires 157.0613. Anal. Calcd. For $\mathrm{C}_{6} \mathrm{H}_{8} \mathrm{~N}_{2} \mathrm{O}_{3}: \mathrm{C}, 46.15 ; \mathrm{H}$, 5.16; N, 17.94. Found: C, 46.46; H, 5.28; N, 18.07 .

\section{Ethyl Cyanodiazoacetate}

Ethyl cyanoacetate $(0.11 \mathrm{~mL}, 1.0 \mathrm{mmol})$ was treated according to Procedure $\mathrm{C}$, using pyridine $(0.40$ $\mathrm{mL}, 5.0 \mathrm{mmol}$ ) as base [flash chromatography (EtOAc/petrol, 1:19)], to give ethyl cyanodiazoacetate as a yellow oil (85 mg, 61\%). The IR, ${ }^{1} \mathrm{H}$ and ${ }^{13} \mathrm{C}$ NMR spectroscopic data were in agreement with those published. ${ }^{13}$ HRMS (FAB): $\mathrm{m} / \mathrm{z}=140.0463 ;[\mathrm{M}+\mathrm{H}]^{+}$requires 140.0460. Anal. Calcd. For $\mathrm{C}_{5} \mathrm{H}_{5} \mathrm{~N}_{3} \mathrm{O}_{2}: \mathrm{C}, 43.17 ; \mathrm{H}, 3.62 ; \mathrm{N}, 30.20$. Found: $\mathrm{C}, 43.15 ; \mathrm{H}, 3.78 ; \mathrm{N}, 30.16$.

(11) Xiong, Y.; Bernardi, D.; Bratton, S.; Ward, M. D.; Battaglia, E.; Finel, M.; Drake, R. R.; Radominska-Pandya, A. Biochemistry 2006, 45, 2322-2332.

(12) Rianelli, R. de S.; de Souza, M. C. B. V.; Ferreira, V. F. Synth. Commun. 2004, 34, 951-959.

(13) Wurz, R. P.; Lin, W.; Charette, A. B. Tetrahedron Lett. 2003, 44, 8845-8848. 


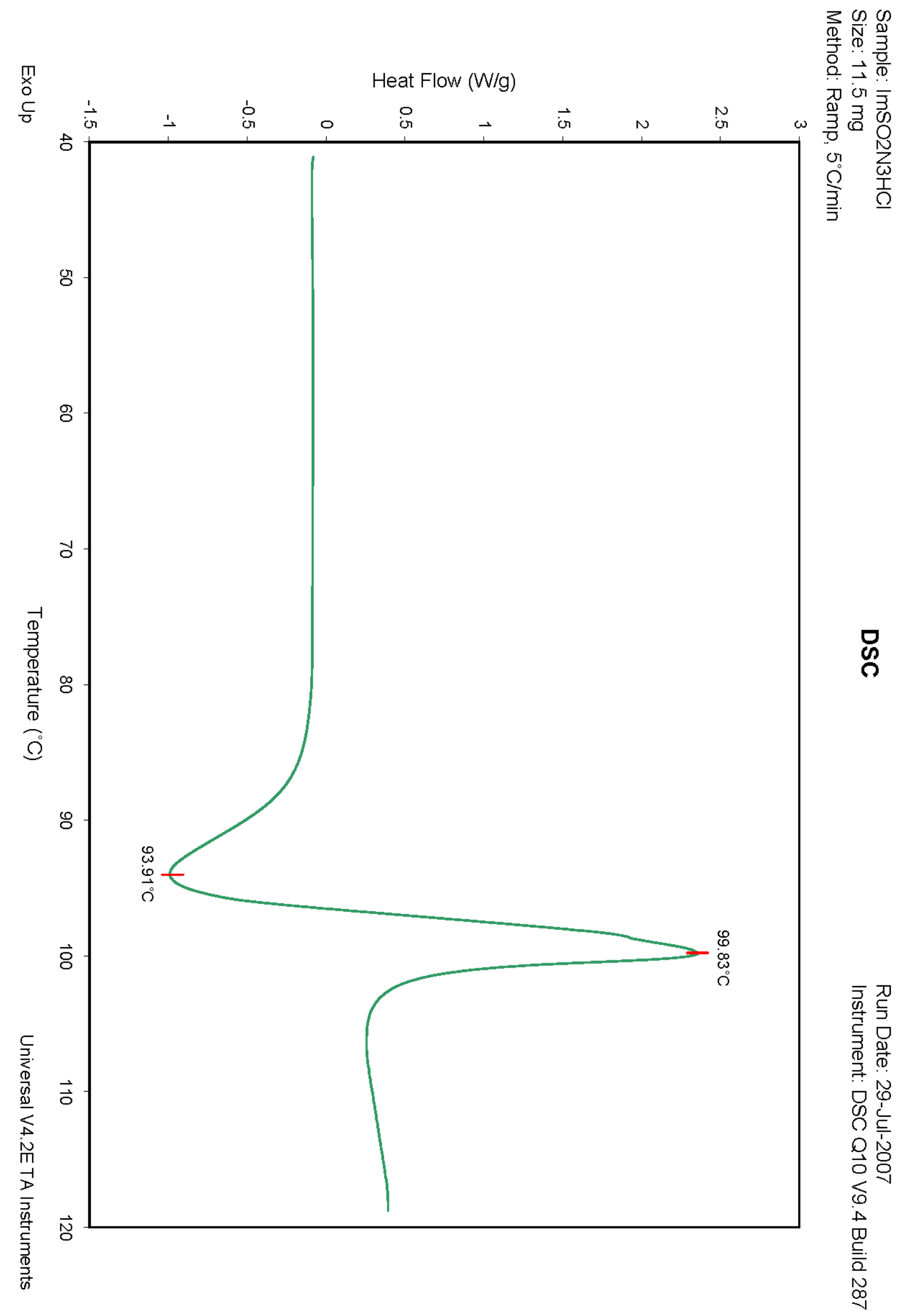


Imidazole-1-sulfonyl Azide 1

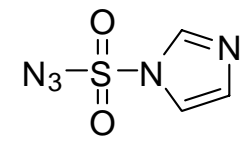

${ }^{1} \mathrm{H}$ NMR (600 MHz, $\mathrm{CDCl}_{3}$ )

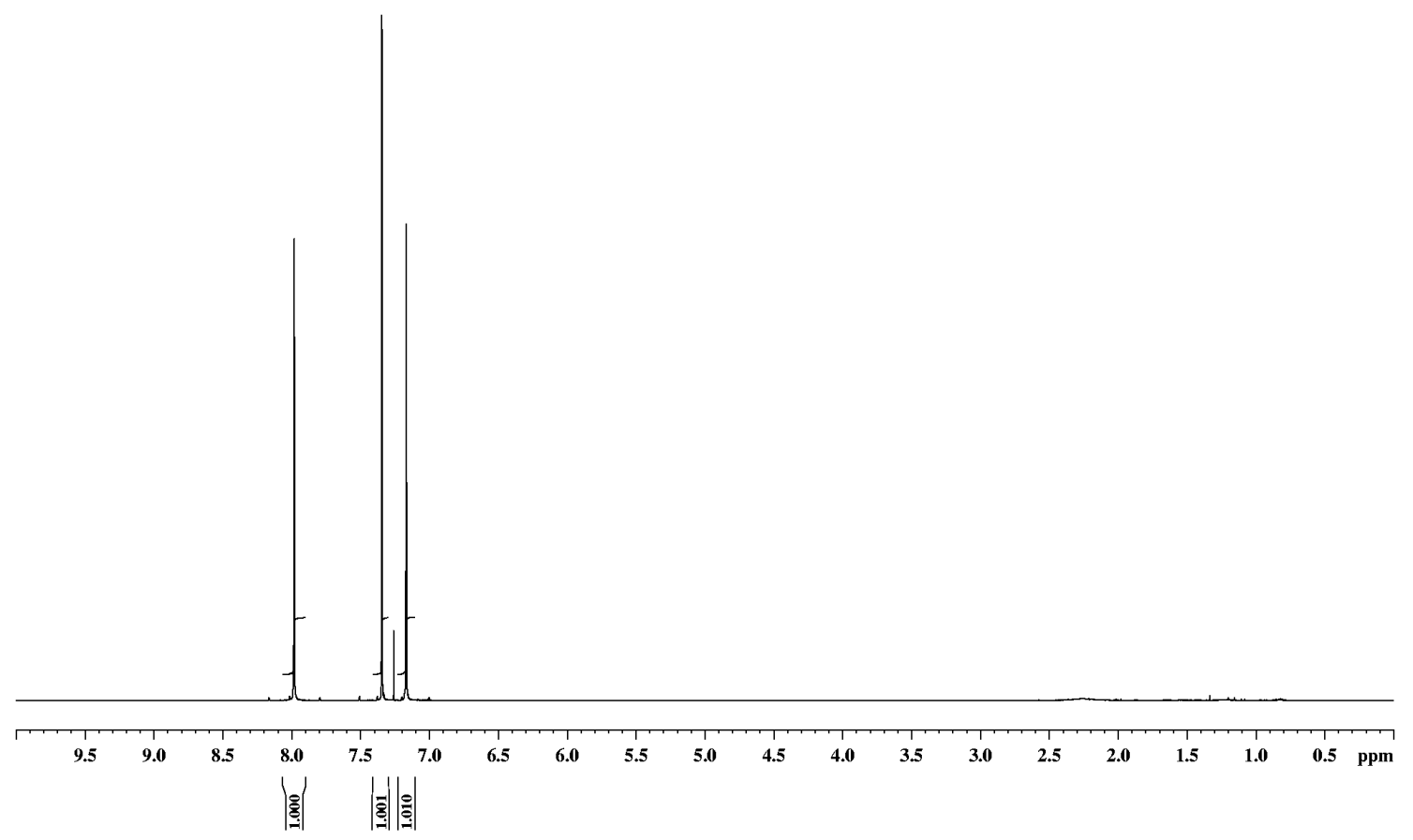

${ }^{13} \mathrm{C}$ NMR (150.9 MHz, $\mathrm{CDCl}_{3}$ )

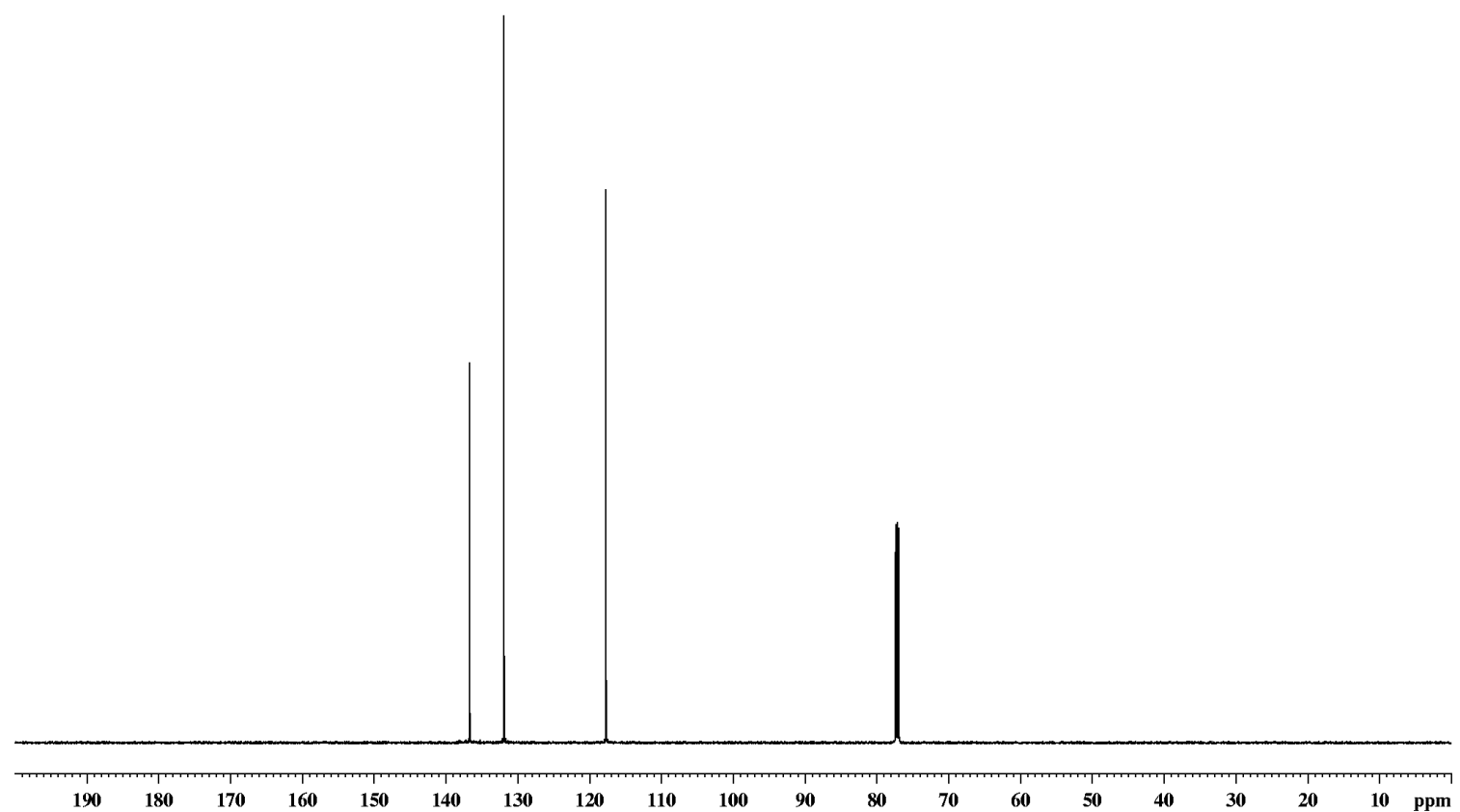


Imidazole-1-sulfonyl Azide Hydrochloride 1.HCl<smiles>NS(=O)(=O)n1ccnc1</smiles>

${ }^{1} \mathrm{H}$ NMR (600 MHz, D $\left.2 \mathrm{O}\right)$

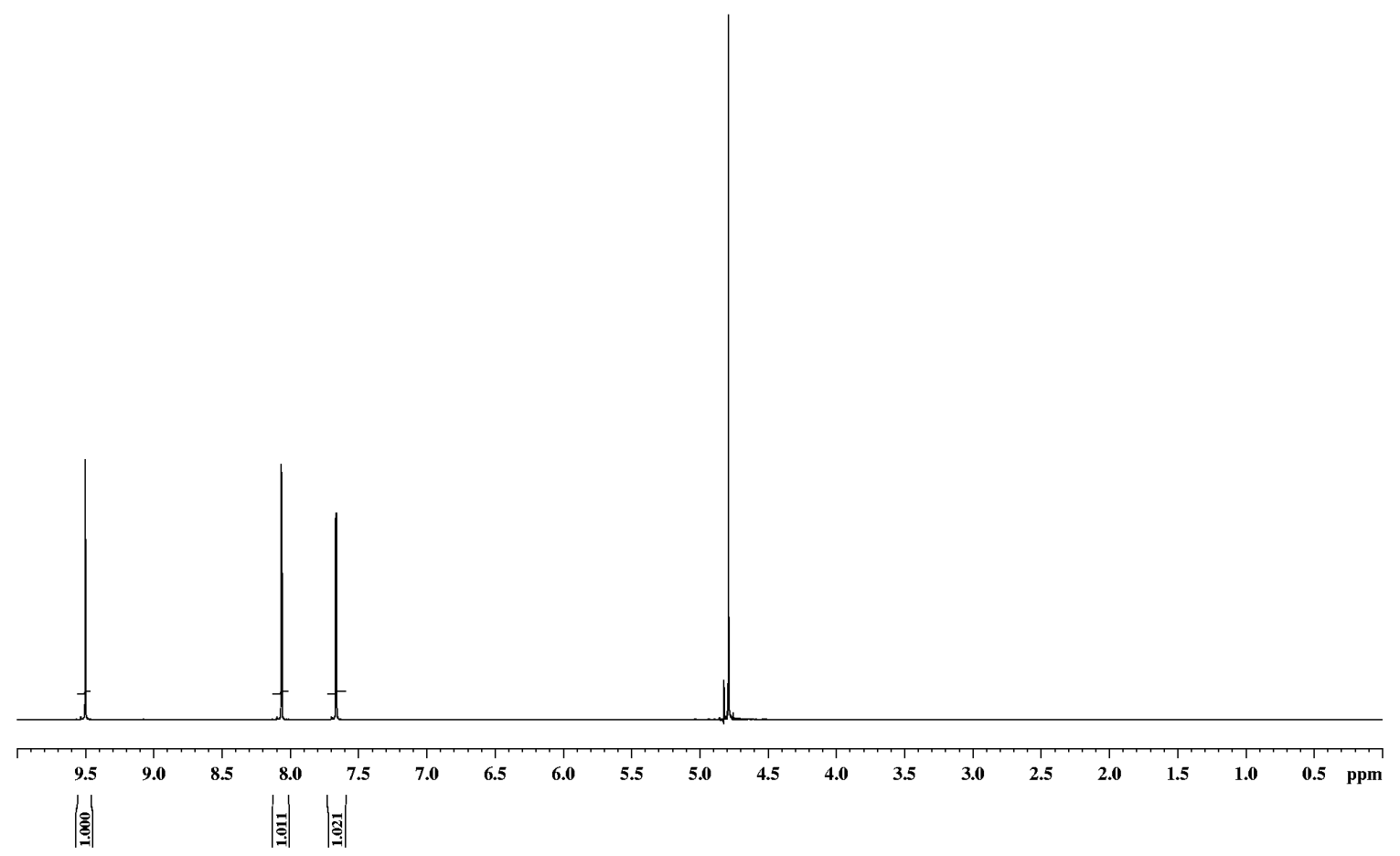

${ }^{13} \mathrm{C}$ NMR (150.9 MHz, $\left.\mathrm{D}_{2} \mathrm{O}\right)$

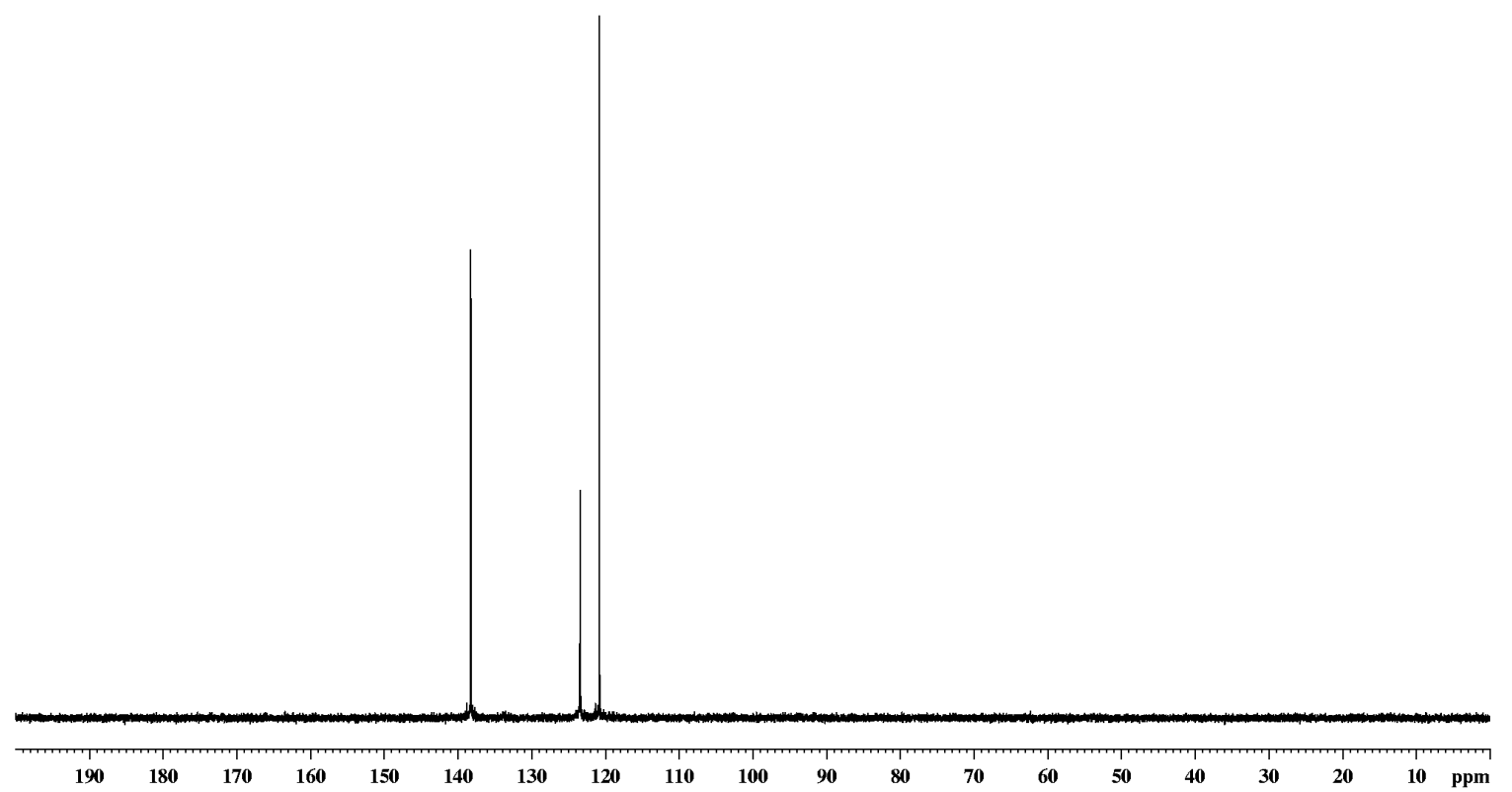


(2S)-2-Azido-3-methylbutanoic acid

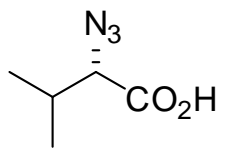

${ }^{1} \mathrm{H} \mathrm{NMR}\left(600 \mathrm{MHz}, \mathrm{CDCl}_{3}\right)$

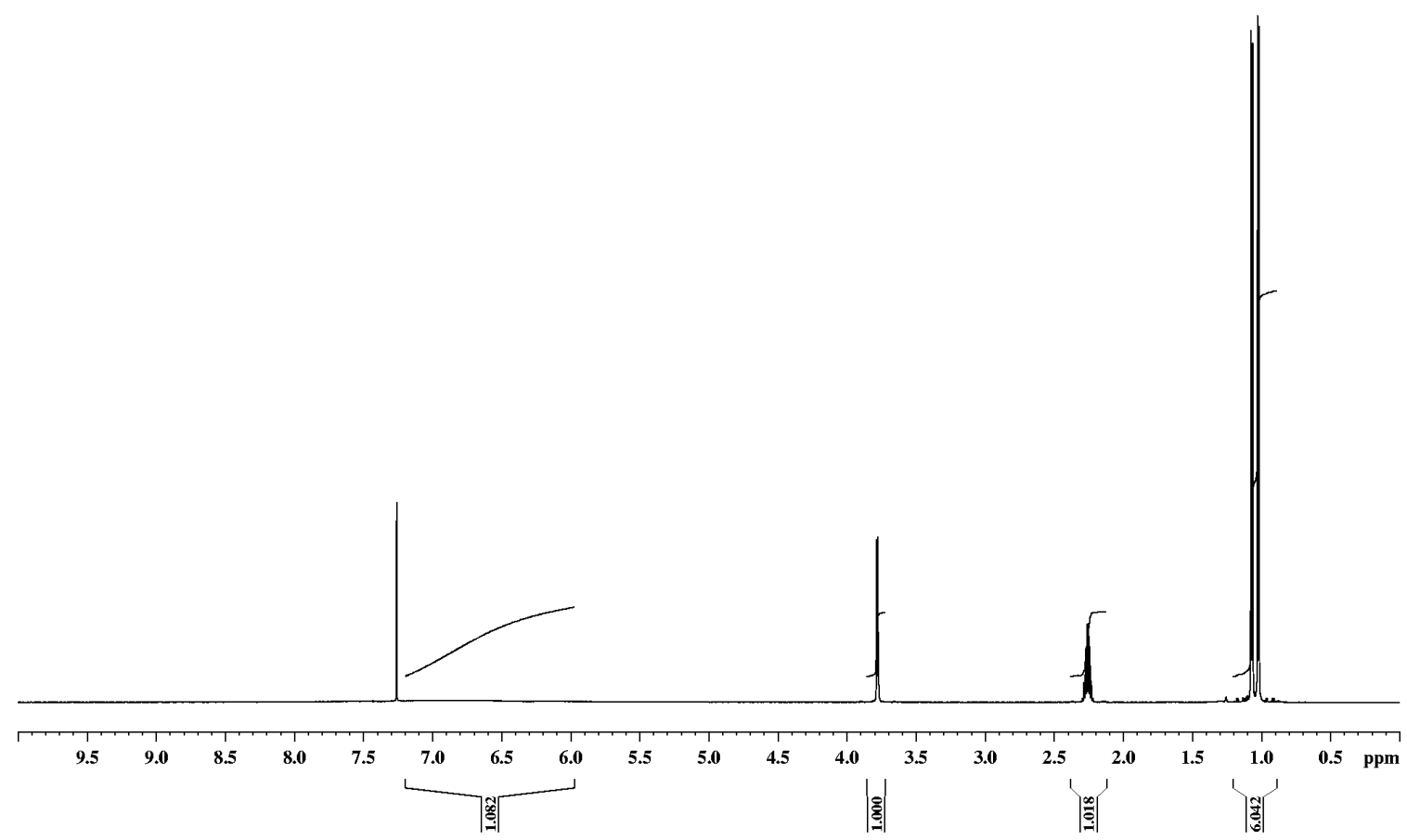

${ }^{13} \mathrm{C}$ NMR (150.9 MHz, $\left.\mathrm{CDCl}_{3}\right)$

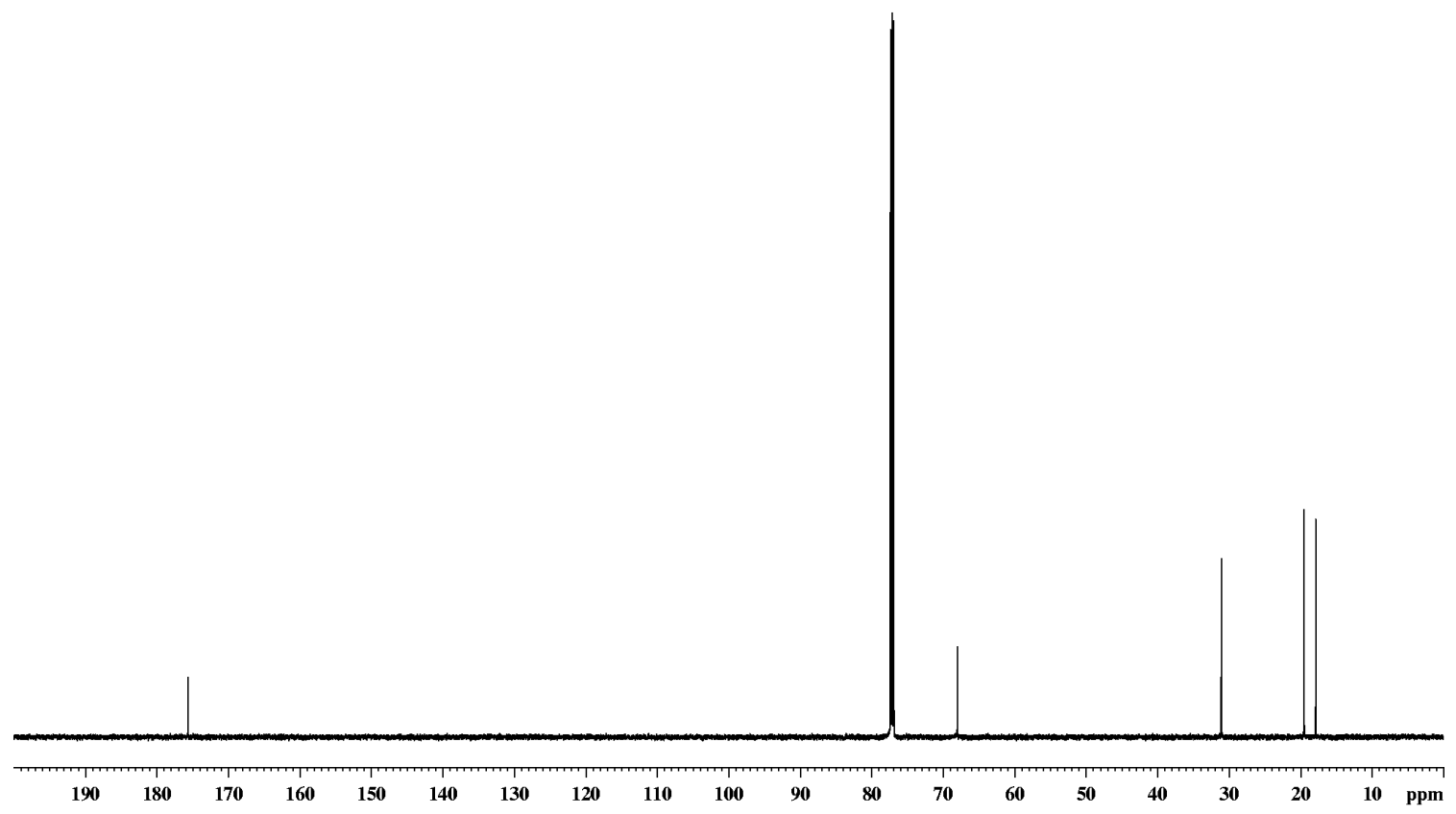




\section{(2S)-2-Azido-4-methylpentanoic acid}

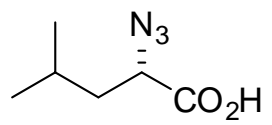

${ }^{1} \mathrm{H}$ NMR $\left(600 \mathrm{MHz}, \mathrm{CDCl}_{3}\right)$

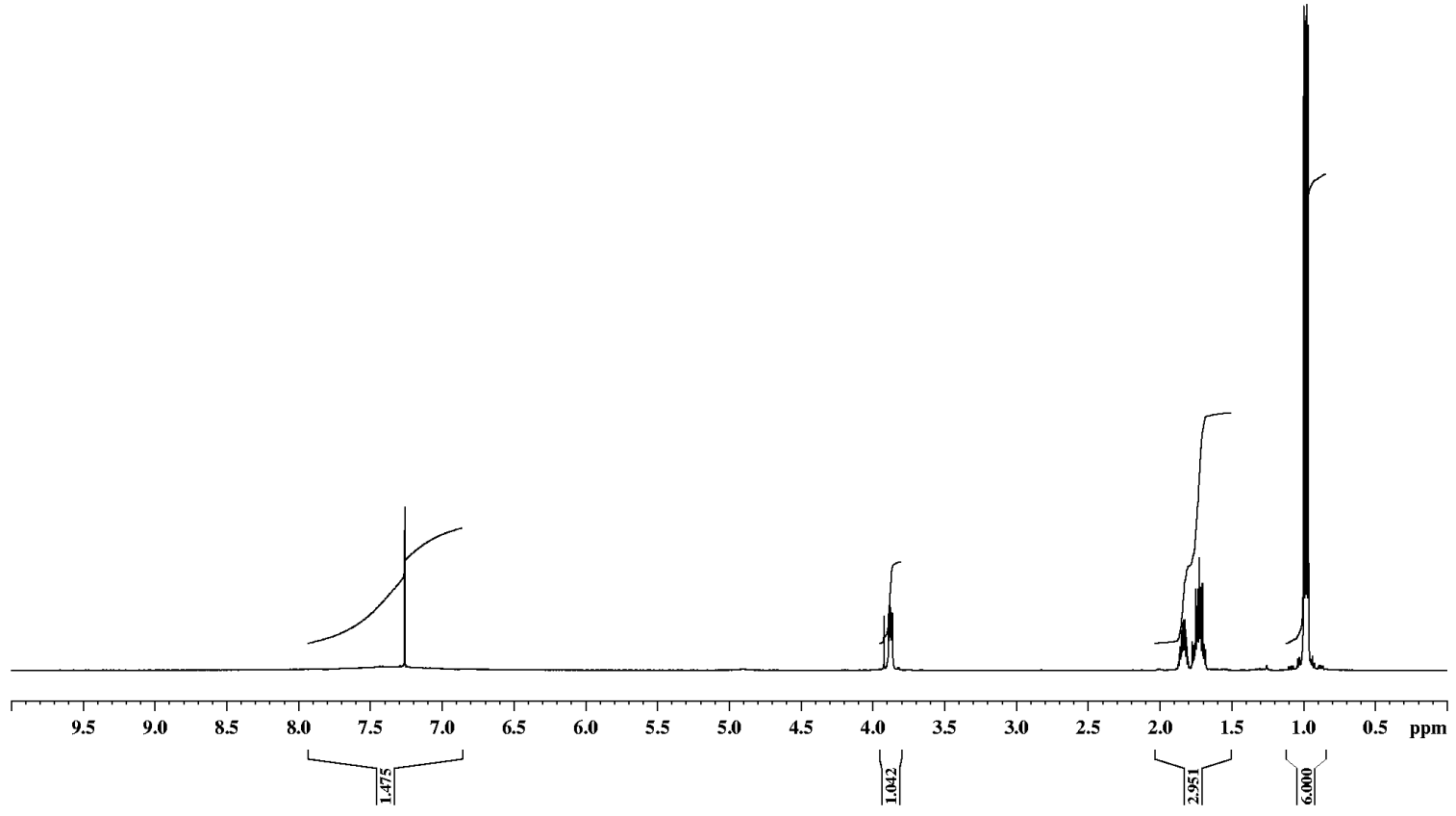

${ }^{13} \mathrm{C}$ NMR $\left(150.9 \mathrm{MHz}, \mathrm{CDCl}_{3}\right)$

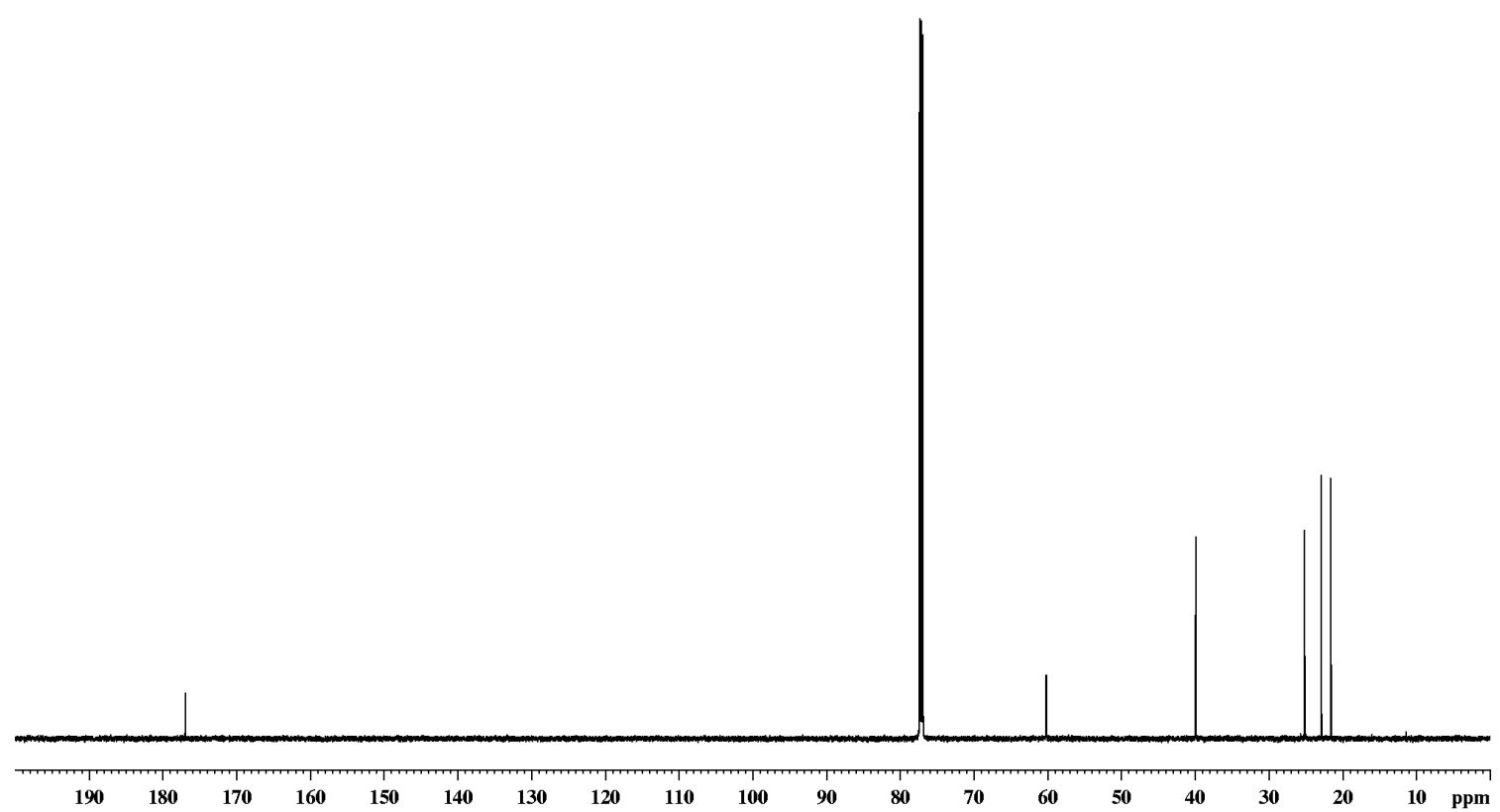


(2S)-2,6-Diazidohexanoic Acid

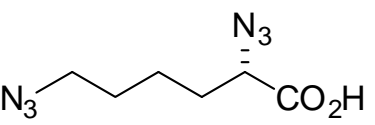

${ }^{1} \mathrm{H}$ NMR (600 MHz, $\mathrm{CDCl}_{3}$ )

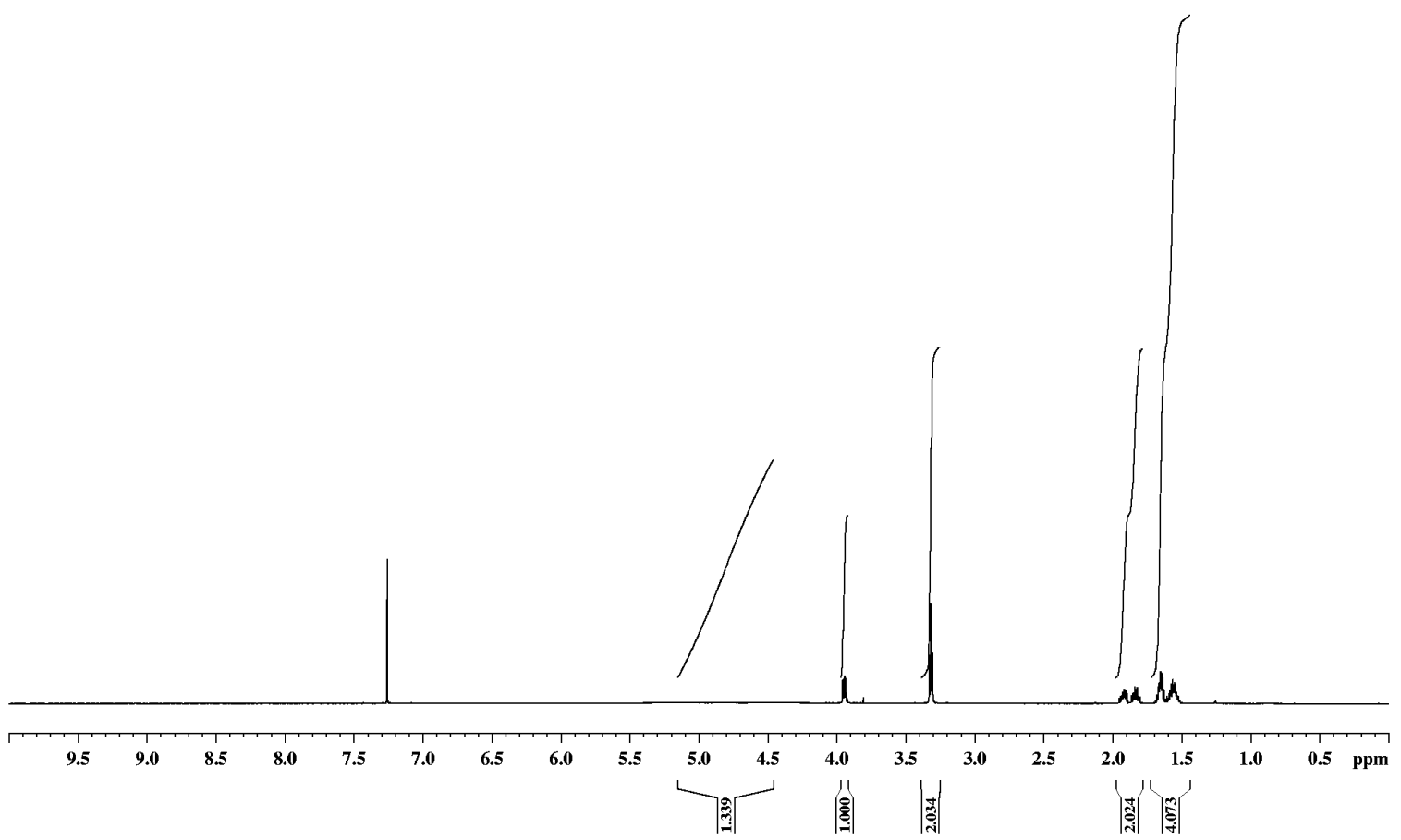

${ }^{13} \mathrm{C}$ NMR (150.9 MHz, $\mathrm{CDCl}_{3}$ )

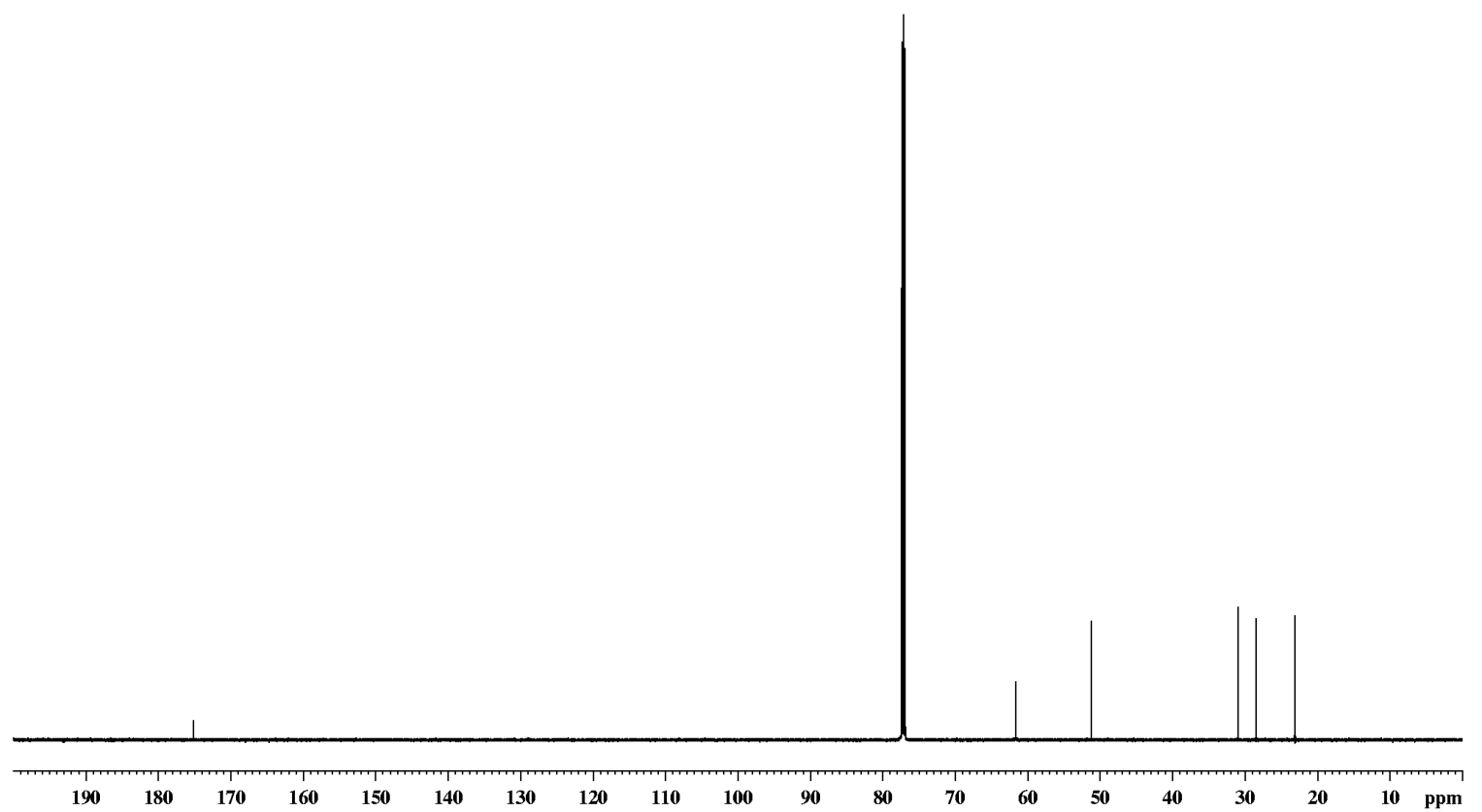




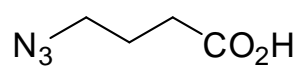

${ }^{1} \mathrm{H}$ NMR (600 MHz, $\mathrm{CDCl}_{3}$ )

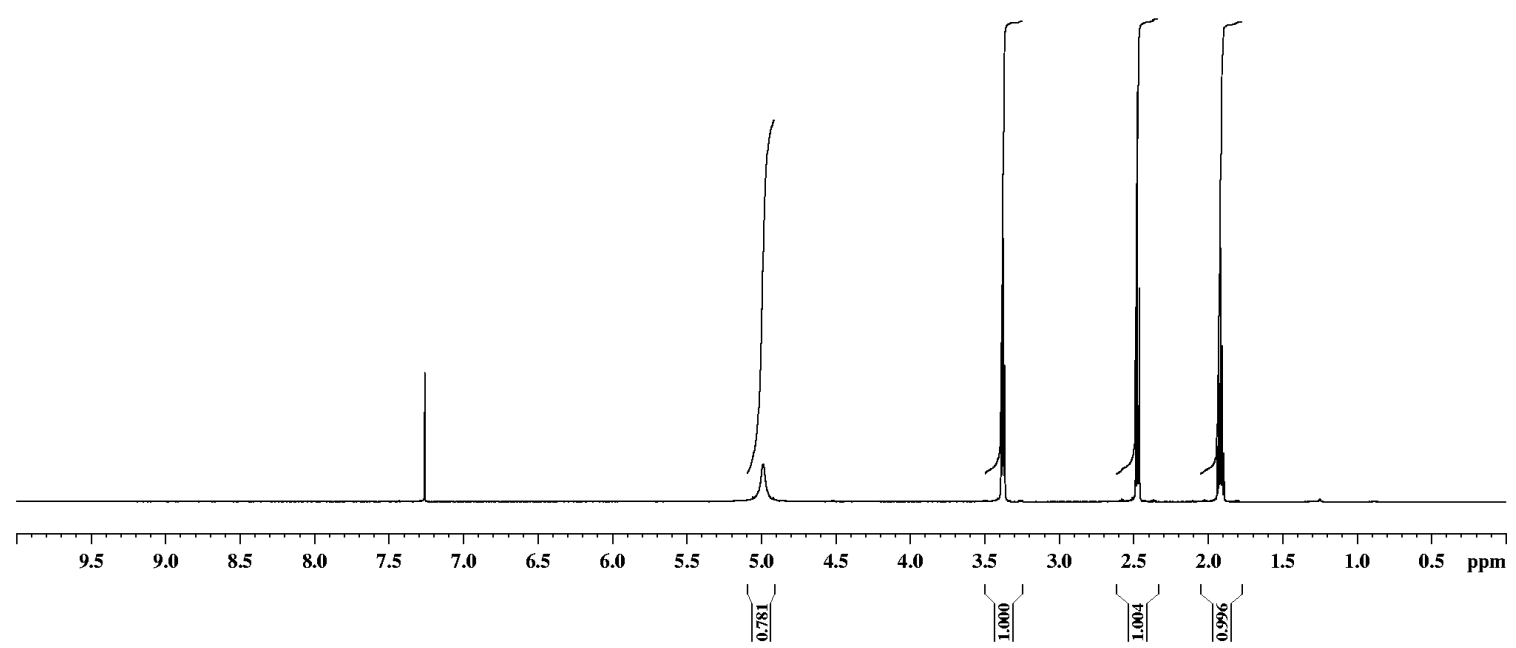

${ }^{13} \mathrm{C}$ NMR (150.9 MHz, $\mathrm{CDCl}_{3}$ )

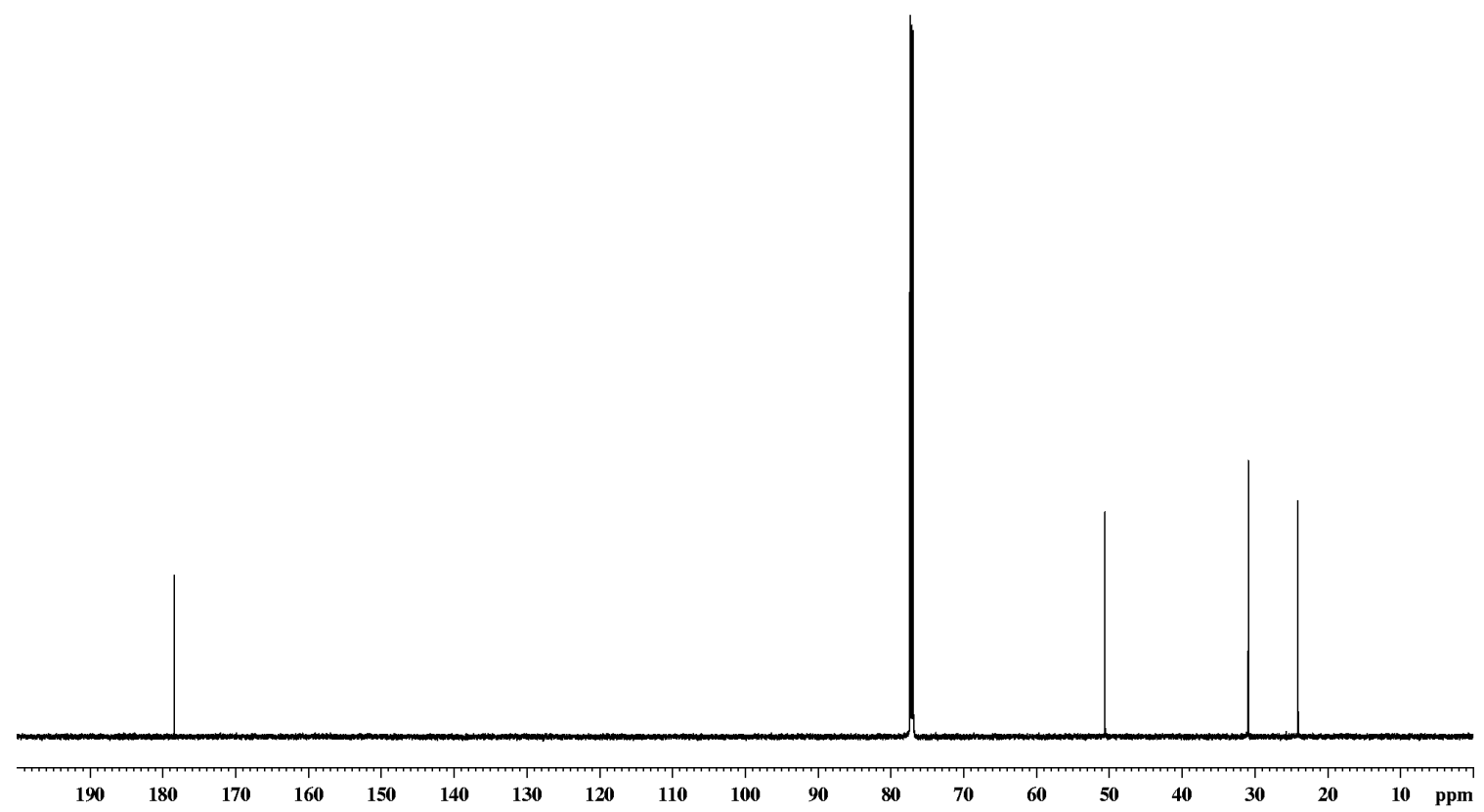


Methyl (2S)-2-Azido-3-(4-hydroxyphenyl)propanoate

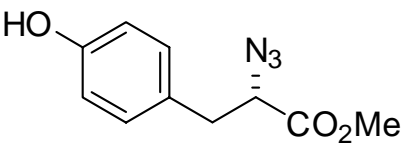

${ }^{1} \mathrm{H} \mathrm{NMR}\left(600 \mathrm{MHz}, \mathrm{CDCl}_{3}\right)$

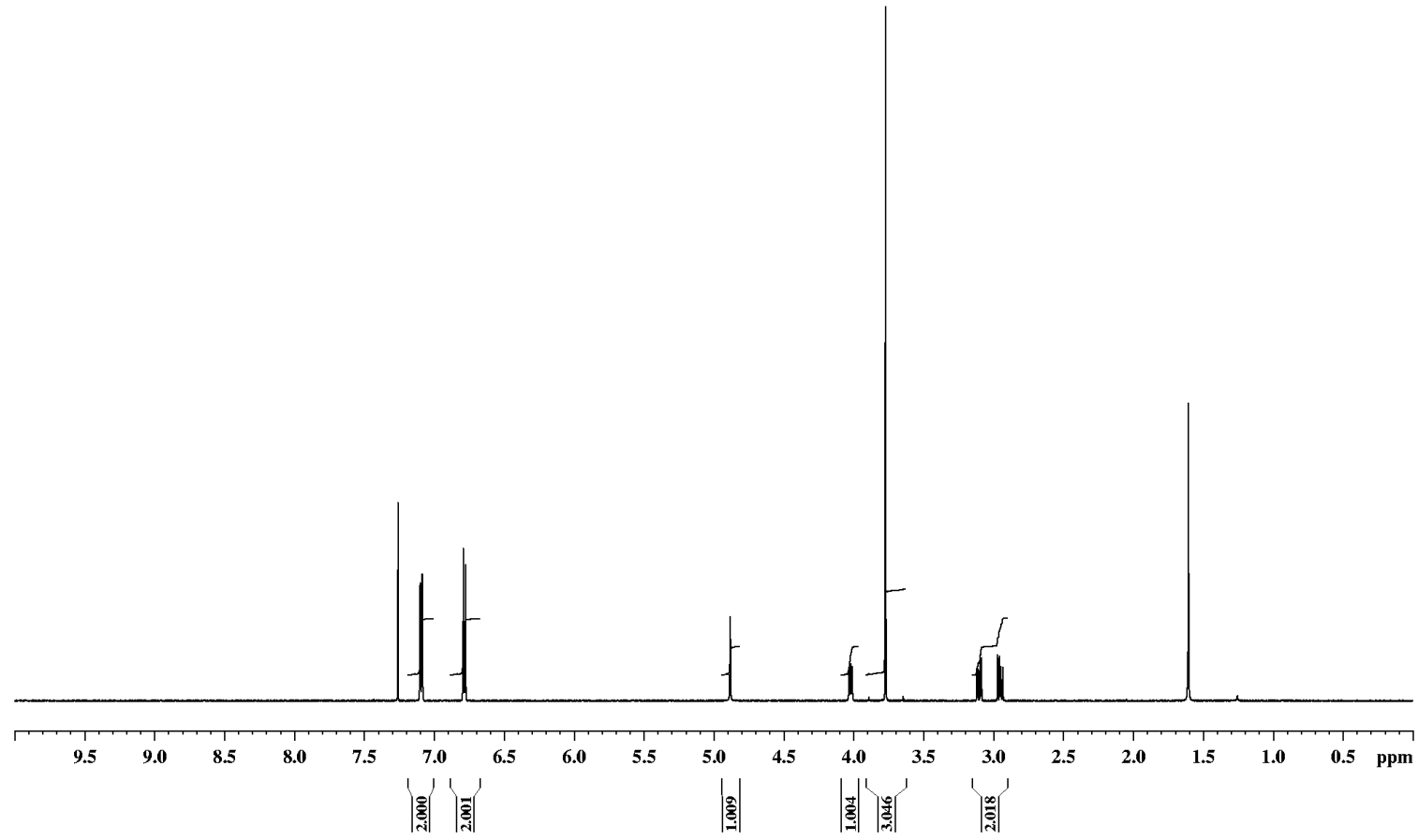

${ }^{13} \mathrm{C}$ NMR (150.9 MHz, $\left.\mathrm{CDCl}_{3}\right)$

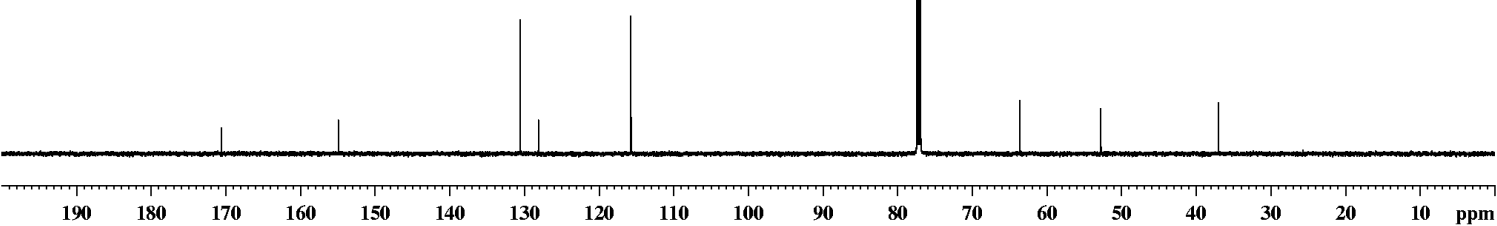


(1S,2S)-2-Azido-1-phenyl-1,3-propanediol<smiles>[15NH][C@H](CO)[C@@H](O)c1ccccc1</smiles>

${ }^{1} \mathrm{H}$ NMR $\left(600 \mathrm{MHz}, \mathrm{CDCl}_{3}\right)$

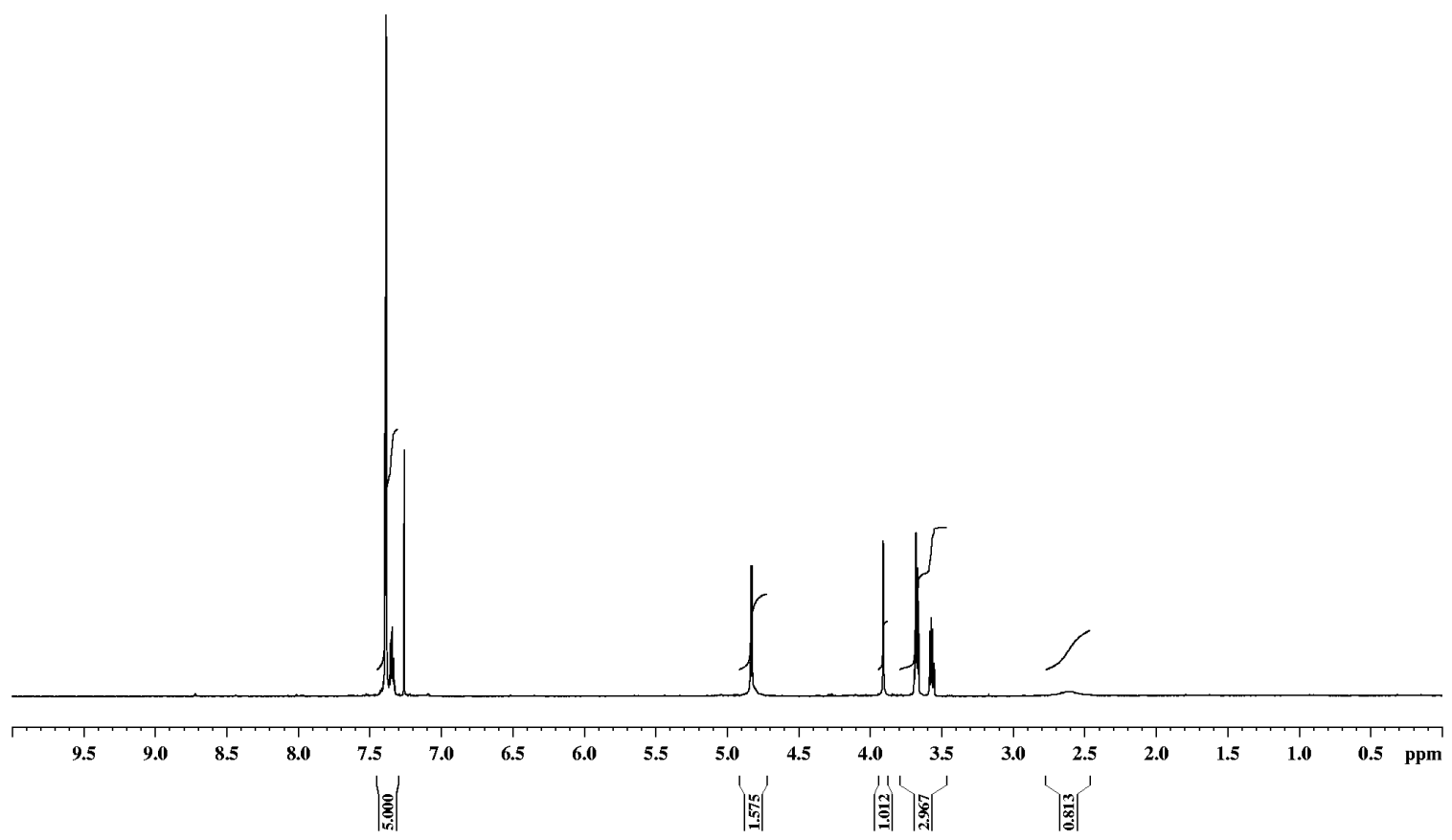

${ }^{13} \mathrm{C}$ NMR (150.9 MHz, $\mathrm{CDCl}_{3}$ )

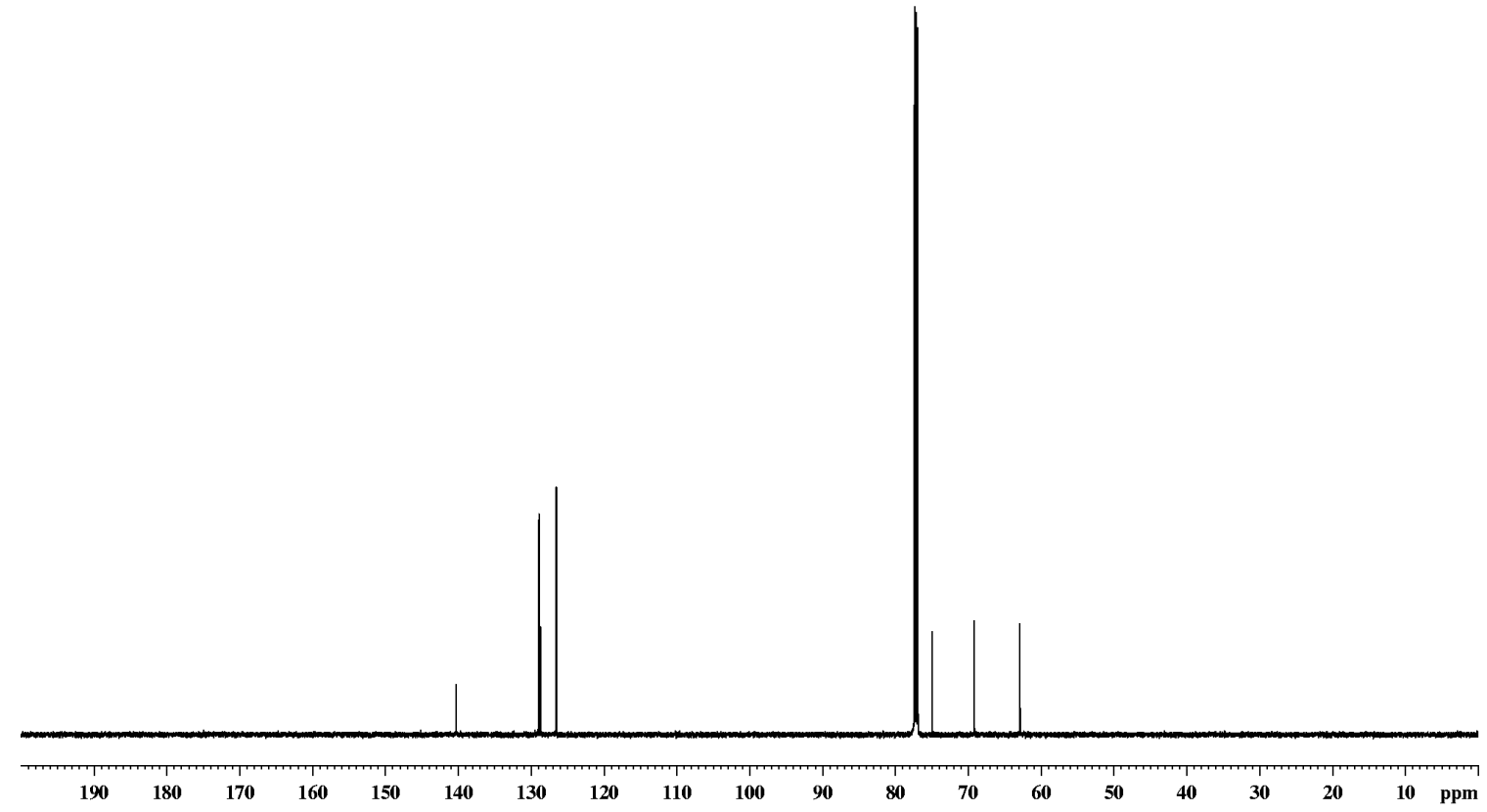


<smiles>COc1ccc([N+]#N)cc1</smiles>

${ }^{1} \mathrm{H}$ NMR $\left(600 \mathrm{MHz}, \mathrm{CDCl}_{3}\right)$

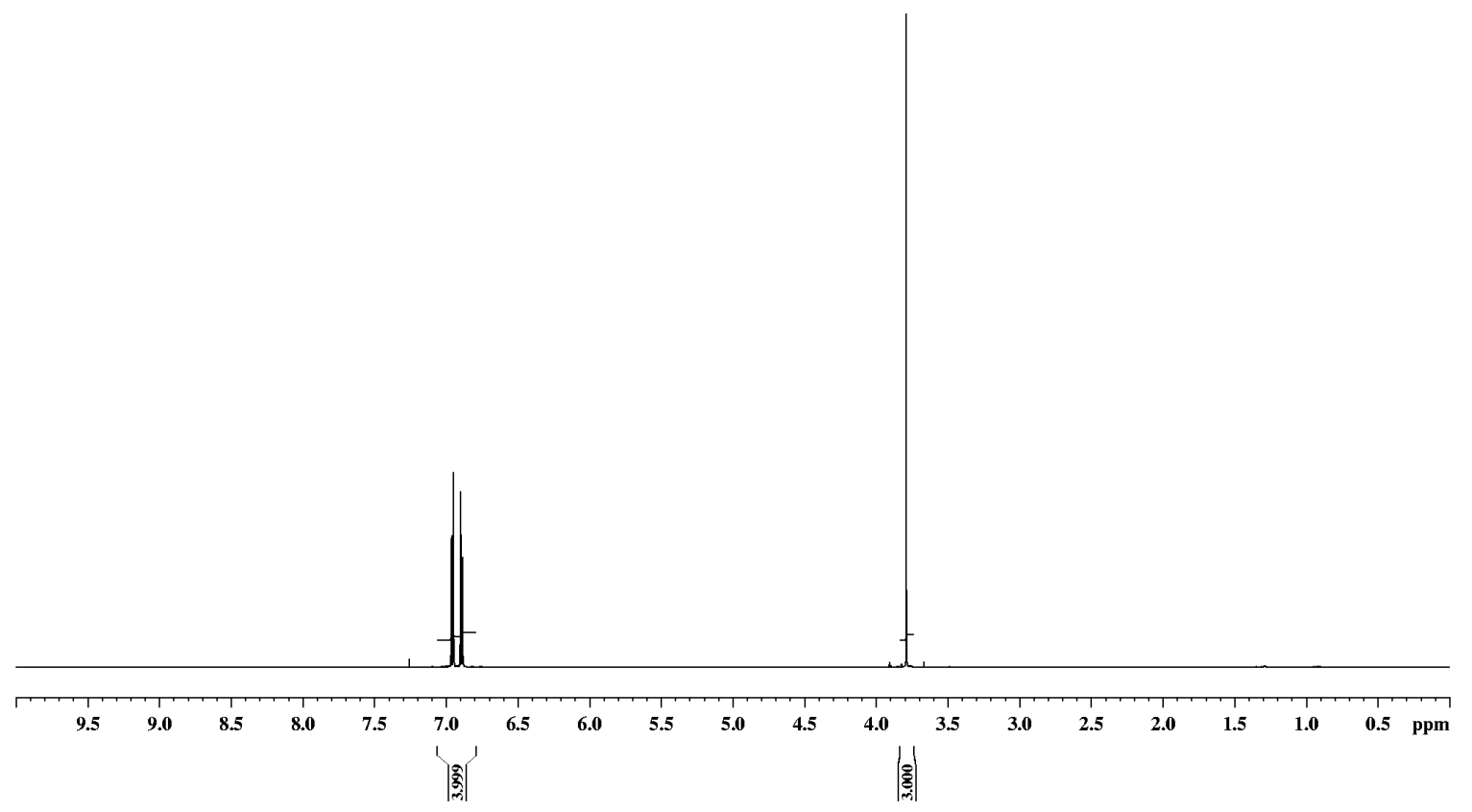

${ }^{13} \mathrm{C}$ NMR (150.9 MHz, $\mathrm{CDCl}_{3}$ )

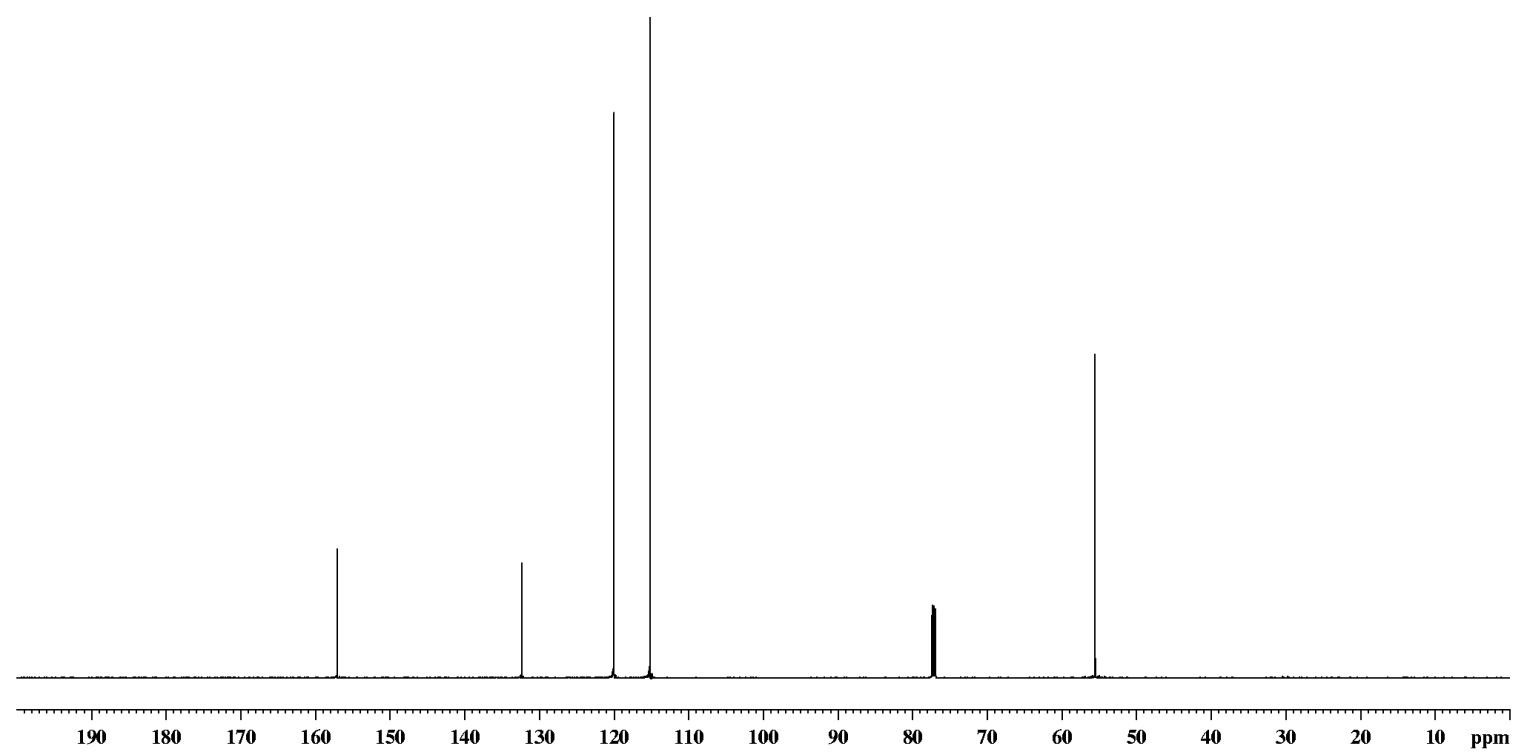




\section{4-Azidobenzoic acid}<smiles>N#[N+]c1ccc(C(=O)O)cc1</smiles>

${ }^{1} \mathrm{H}$ NMR $\left(600 \mathrm{MHz},\left(\mathrm{CD}_{3}\right)_{2} \mathrm{SO}\right)$

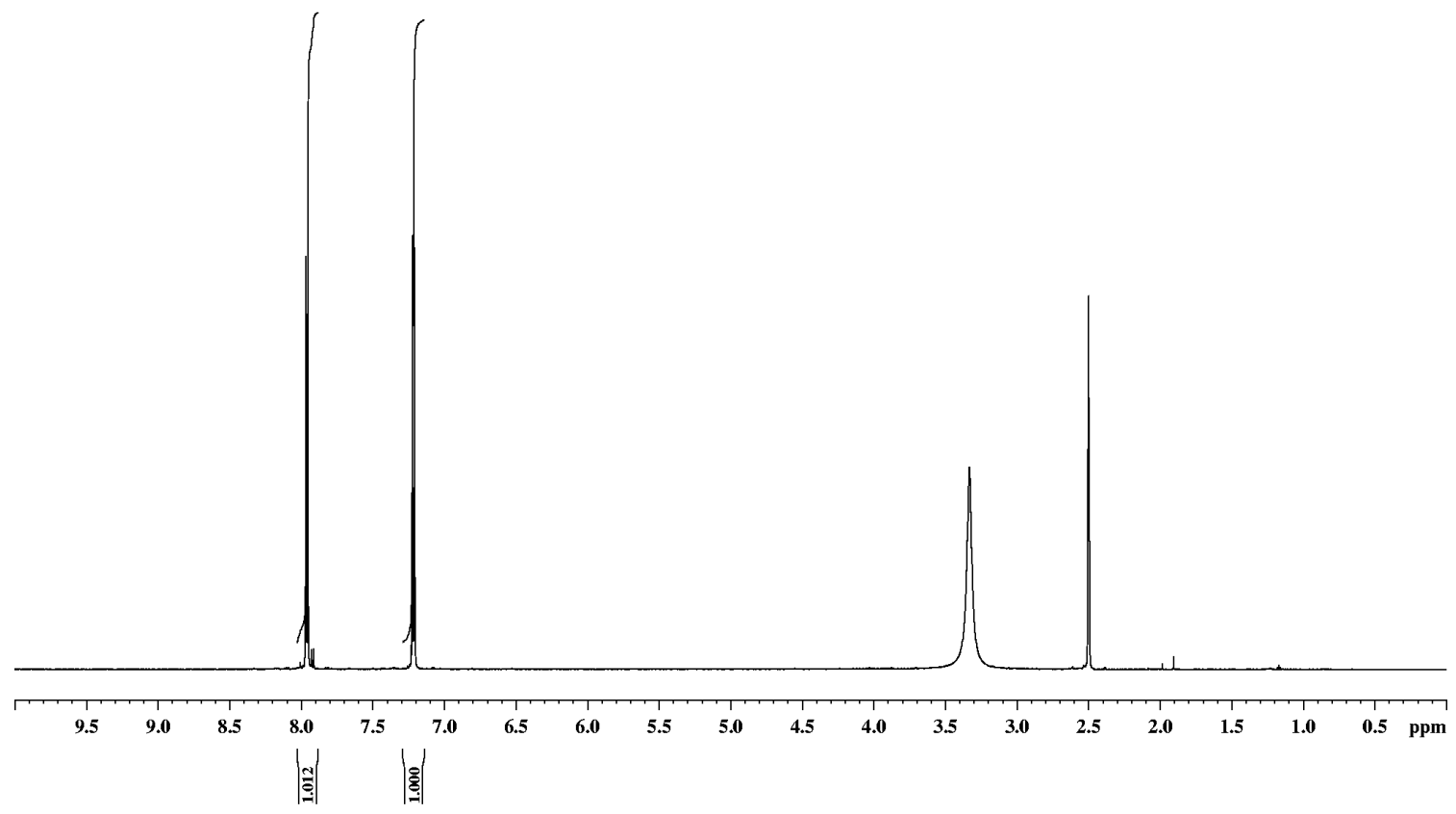

${ }^{13} \mathrm{C}$ NMR $\left(150.9 \mathrm{MHz},\left(\mathrm{CD}_{3}\right)_{2} \mathrm{SO}\right)$

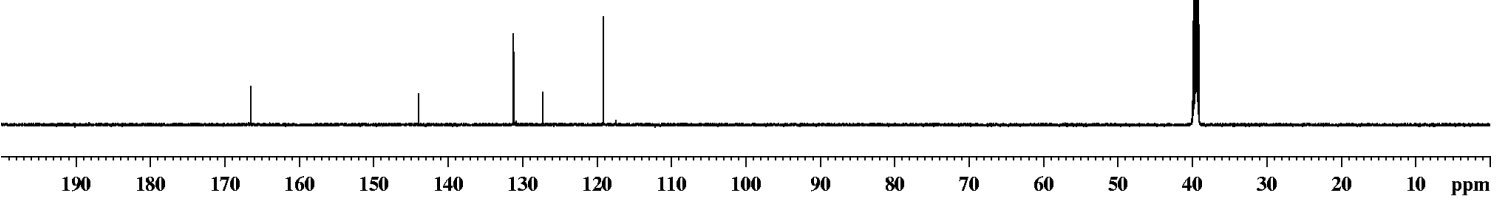




\section{2-Azidobenzoic acid}<smiles>Nc1ccccc1C(=O)O</smiles>

${ }^{1} \mathrm{H}$ NMR $\left(600 \mathrm{MHz}, \mathrm{CDCl}_{3}\right)$

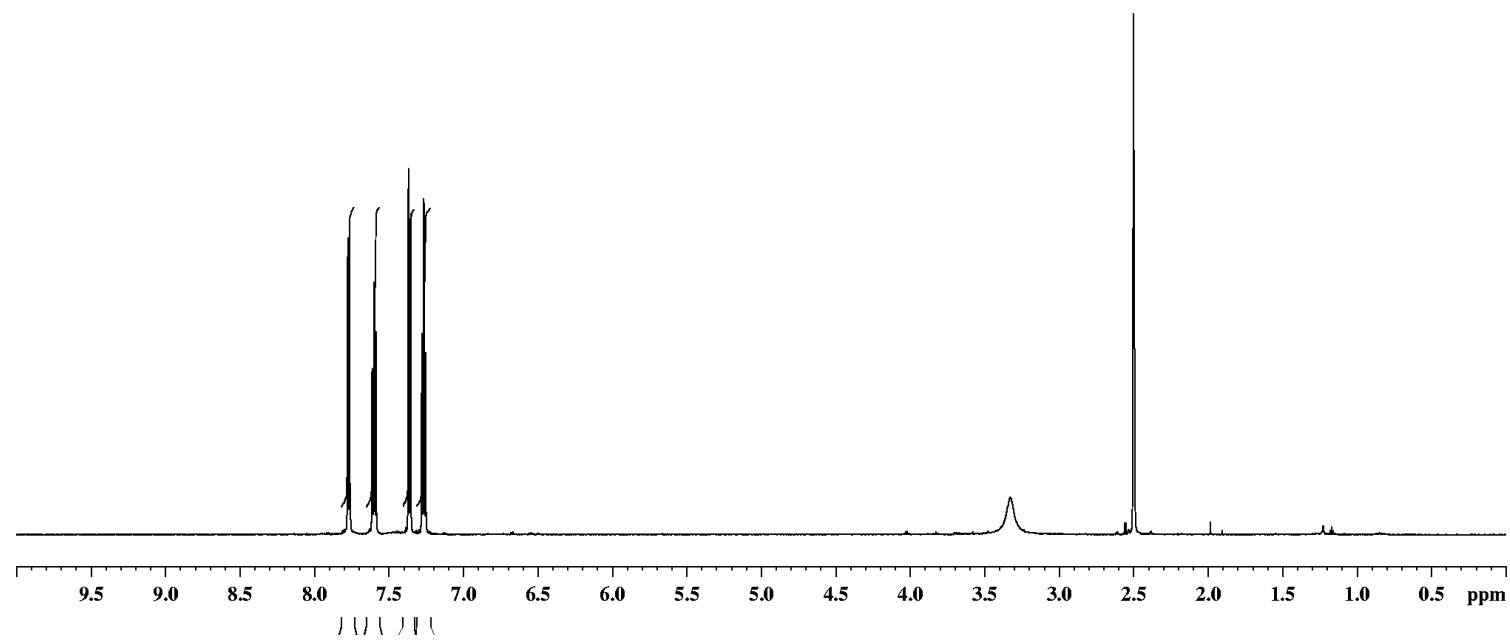

${ }^{13} \mathrm{C}$ NMR (150.9 MHz, $\mathrm{CDCl}_{3}$ )

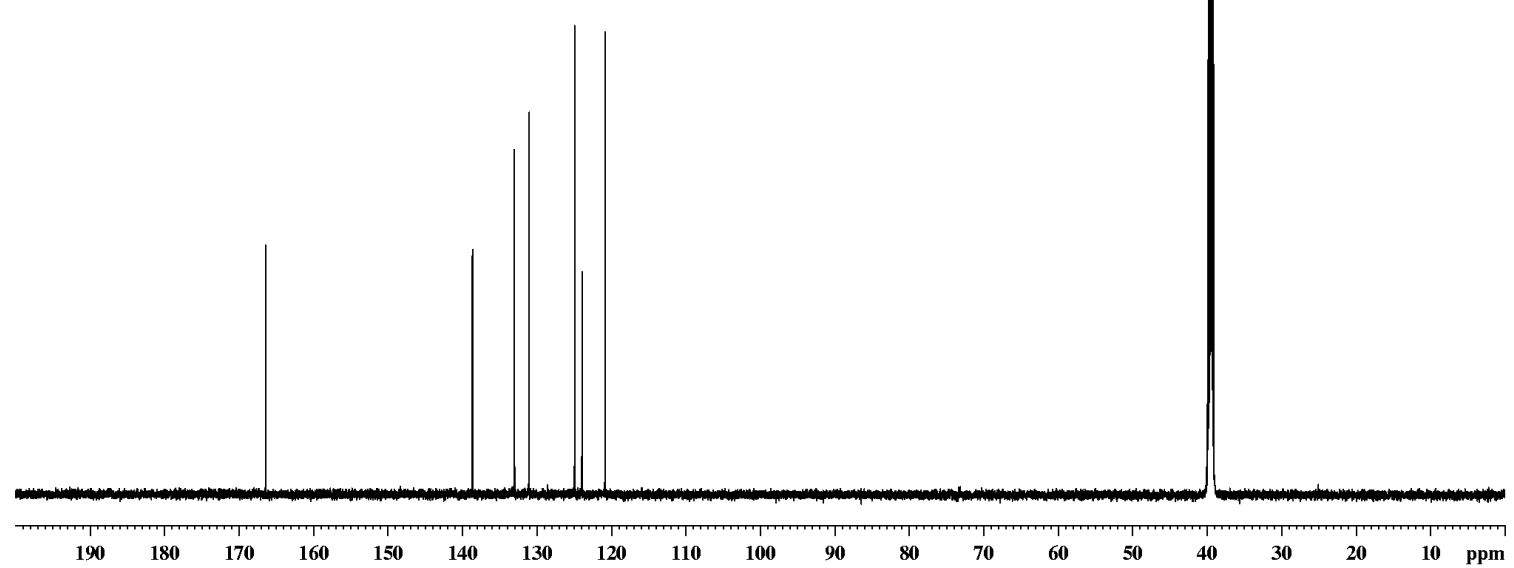

